\title{
Identifying the Role of Complement in Triggering Neuroinflammation after Traumatic Brain Injury
}

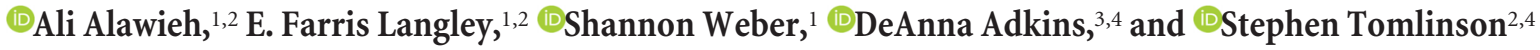 \\ ${ }^{1}$ Medical Scientist Training Program and Departments of, ${ }^{2}$ Microbiology and Immunology, ${ }^{3}$ Neurosciences, Medical University of South Carolina, \\ Charleston, South Carolina 29425, and 4Ralph Johnson VA Medical Center, Charleston, South Carolina 29401
}

The complement system is implicated in promoting acute secondary injury after traumatic brain injury (TBI), but its role in chronic post-traumatic neuropathology remains unclear. Using various injury-site targeted complement inhibitors that block different complement pathways and activation products, we investigated how complement is involved in neurodegeneration and chronic neuroinflammation after TBI in a clinically relevant setting of complement inhibition. The current paradigm is that complement propagates post-TBI neuropathology predominantly through the terminal membrane attack complex (MAC), but the focus has been on acute outcomes. Following controlled cortical impact in adult male mice, we demonstrate that although inhibition of the MAC (with CR2-CD59) reduces acute deficits, inhibition of $\mathrm{C} 3$ activation is required to prevent chronic inflammation and ongoing neuronal loss. Activation of C3 triggered a sustained degenerative mechanism of microglial and astrocyte activation, reduced dendritic and synaptic density, and inhibited neuroblast migration several weeks after TBI. Moreover, inhibiting all complement pathways (with CR2-Crry), or only the alternative complement pathway (with CR2-fH), provided similar and significant improvements in chronic histological, cognitive, and functional recovery, indicating a key role for the alternative pathway in propagating chronic post-TBI pathology. Although we confirm a role for the MAC in acute neuronal loss after TBI, this study shows that upstream products of complement activation generated predominantly via the alternative pathway propagate chronic neuroinflammation, thus challenging the current concept that the MAC represents a therapeutic target for treating TBI. A humanized version of CR2fH has been shown to be safe and non-immunogenic in clinical trials.

Key words: chronic injury; complement; inflammation; therapy

Significance Statement

Complement, and specifically the terminal membrane attack complex, has been implicated in secondary injury and neuronal loss after TBI. However, we demonstrate here that upstream complement activation products, generated predominantly via the alternative pathway, are responsible for propagating chronic inflammation and injury following CCI. Chronic inflammatory microgliosis is triggered by sustained complement activation after CCI, and is associated with chronic loss of neurons, dendrites and synapses, a process that continues to occur even $30 \mathrm{~d}$ after initial impact. Acute and injury-site targeted inhibition of the alternative pathway significantly improves chronic outcomes, and together these findings modify the conceptual paradigm for targeting the complement system to treat TBI.

\section{Introduction}

Traumatic brain injury (TBI) results in a complex multiphasic pathophysiological response that exacerbates the initial injury

Received Aug. 3, 2017; revised Jan. 3, 2018; accepted Jan. 29, 2018.

Author contributions: A.A. and S.T. designed research;A.A., E.F.L., and S.W. performed research; D.A. contributed unpublished reagents/analytic tools; A.A., E.F.L., S.W., D.A., and S.T. analyzed data; A.A. and S.T. wrote the paper.

This work was supported by Grants from the NIH (1P20GM109040, U54GM10494, U54GM10491) to D.A. and S.T., the Department of Veterans Affairs (Merit Award 1101RX001141 and 1121RX002363) to S.T., and an American Heart Association Predoctoral Fellowship to A.A. (15PRE25250009); and the use of the MUSC Cell and Molecular Imaging Core (supported by awards from the NIH P30 CA138313 and S10 0D018113), the Center for Oral Health Research (NIGMS Award P30GM103331), and the Josh Spruill Imaging Facility (NIH-NIGMS P30 GM103342).

Conflict of interest: Stephen Tomlinson is an inventor on a licensed patent for CR2-targeted complement inhibition. The remaining authors declare no competing financial interests.

Correspondence should be addressed to Dr. Stephen Tomlinson, Department of Microbiology and Immunology, 173 Ashley Avenue, BSB 204, MSC 504, Charleston, SC 29425. E-mail: tomlinss@musc.edu. and restricts acute and chronic recovery (Blennow et al., 2012; Kumar and Loane, 2012). Inflammation is thought to be one component of the progressive degenerative events that take place after TBI, and the complement system has been recognized as one contributor to inflammation and secondary insult through promoting neuronal loss, edema, and inflammatory cellular infiltrate (Kaczorowski et al., 1995; Hicks et al., 2002; Rancan et al., 2003; Sewell et al., 2004; Leinhase et al., 2006a,b, 2007; Longhi et al., 2009; Stahel et al., 2009; Neher et al., 2011; Fluiter et al., 2014; Ruseva et al., 2015; Rich et al., 2016). Clinical studies have demonstrated that TBI patients have elevated levels of complement activation prod- 
ucts in the CSF and increased deposition of complement activation products in the perilesional brain (Kossmann et al., 1997; Stahel et al., 1998, 2001; Bellander et al., 2001, 2011).

Complement can be activated via the classical, lectin or alternative pathway, with the alternative complement pathway (ACP) also serving as an amplification loop. Complement activation leads to the deposition of opsonins, generation of anaphylatoxins and assembly of the terminal membrane attack complex (MAC). Nearly all studies in preclinical models have investigated how complement deficiency or inhibition affects acute outcomes after TBI (Kaczorowski et al., 1995; Kossmann et al., 1997; Stahel et al., 1998, 2001; Bellander et al., 2001, 2011; Hicks et al., 2002; Leinhase et al., 2006a, 2007; Ruseva et al., 2015; Rich et al., 2016), and current evidence implicates the MAC as a major mediator of damage (Leinhase et al., 2006a, 2007; Stahel et al., 2009; Fluiter et al., 2014; Ruseva et al., 2015). However, the role of complement and of the $\mathrm{MAC}$ in chronic pathology and outcomes remains unclear.

A limitation of previous work investigating the role of the MAC in TBI is the lack of assessment of outcomes beyond the first week of injury. Also, the previous focus on acute gross motor recovery using clinical scores limits the ability to assess key hallmarks of the disease, including cognitive deficits (Leinhase et al., 2006a, 2007; Stahel et al., 2009; Ruseva et al., 2015). We have recently demonstrated that although transient inhibition of complement locally at the site of injury after stroke significantly improves acute and subacute outcomes, deficiency in complement C3 does not result in sustained protection beyond the acute phase and is associated with suppression of neurogenesis (Alawieh et al., 2015a,b). Here, we investigated the role of complement in long-term outcomes after TBI in a clinically relevant paradigm of complement inhibition using a panel of complement inhibitors linked to CR2. The CR2 moiety targets the complement inhibitor to sites of complement activation and C3d deposition (Song et al., 2003; Atkinson et al., 2005). The complement inhibitors used were as follows: Crry (inhibits all pathways at $\mathrm{C} 3$ activation), factor $\mathrm{H}$ ( $\mathrm{fH}$; inhibits the ACP), and CD59 (inhibits the MAC; Song et al., 2003; Atkinson et al., 2005; Huang et al., 2008; Marshall et al., 2014). Using the murine model of controlled cortical impact (CCI), we demonstrate that although inhibition of the MAC is sufficient to achieve acute neuroprotection, it is not sufficient to prevent chronic neurodegenerative inflammation. We present evidence implicating upstream complement components generated via the ACP in promoting chronic activation of astrocytes and microglia, with neuronal loss occurring chronically with associated cognitive and functional deficits. While acknowledging the limitations of preclinical models of TBI, including the CCI model, our data challenge the previously held concept that the MAC represents a therapeutic target for TBI. Data show that specifically inhibiting the ACP, a less immunosuppressive approach than inhibiting all complement pathways, prevents both acute and chronic neurodegeneration following CCI without interrupting regenerative and reparatory processes.

\section{Materials and Methods}

Statistics. Statistical analyses were performed using GraphPad Prism 6.0. Parametric testing was used unless otherwise specified. Group analyses were performed using one-way ANOVA (or two-way ANOVA for multiple time points) followed by multiple-comparison analyses, repeatedmeasures ANOVA for matched data. $P$ values $<0.05$ were considered significant. Student's $t$ test was used to compare two groups and was always used as two-tailed. Pearson correlation coefficients were used to compute correlations. Data are reported as mean \pm SEM unless otherwise specified. Sample size was estimated based on an effect size determined for each outcome measure by pilot and prior studies. $\mathrm{G}^{\star}$ Power 3
(Universität Düsseldorf, Germany) was used to compute sample size using an acceptable power range of $80-90 \%$. Before surgery, animals were randomly assigned to treatment groups and a double-blinded strategy was used in scoring and assessment thereafter. Treatment groups were coded for each animal and was not accessible to surgeons and investigators performing outcome assessment. Behavioral assessments (open field, anxiety, and Barnes maze) were scored using automated devices or video-analysis tools. Details for statistical analyses for each figure are provided here:

Figure 1a: two-way ANOVA was used with Bonferroni test for multiple comparisons. Overall effect comparing the four different treatment groups was computed. ANOVA statistics: $F_{(3,108)}=10.28, p<0.0001$. All combinations were compared using Bonferroni test for multiple comparisons. Vehicle versus CR2Crry: $p<0.0001$; Vehicle versus CR2fH: $p<0.001$; Vehicle versus CR2CD59: $p<0.01$. Figure $1 b$ : one-way ANOVA was used with Bonferroni test for multiple comparisons. Brown-Forsythe test for homogeneity of variance was not significant: $F_{(3,49)}=0.6546$; $p=$ 0.5839 . ANOVA statistics: $F_{(3,49)}=5.72, p=0.0019$. All combinations were compared using Bonferroni test for multiple comparisons. Vehicle versus CR2Crry: $p<0.001$; Vehicle versus CR2fH: $p<0.05$; Vehicle versus CR2CD59: $p<0.05$. Figure 1c: one-way ANOVA was used with Bonferroni test for multiple comparisons. Brown-Forsythe test for homogeneity of variance was significant: $F_{(3,49)}=7.249 ; p=0.004$. ANOVA statistics: $F_{(3,49)}=0.1374, p=0.9372$. Due to a significant BrownForsythe test, groups were also analyzed using the Kruskal-Wallis (KW) test (nonparametric) showing no significant difference (KW $=0.4503$, $p=0.9297$ ). Figure $1 d$ : survival was analyzed using the log rank (MantelCox) test; $\chi^{2}=2.368 ; p=0.4997$. No significant differences were observed. Figure $2 a-c$ : one-way ANOVA was used with Bonferroni test for multiple comparisons; $a$ : Brown-Forsythe test for homogeneity of variance was not significant: $F_{(3,49)}=1.004 ; p=0.3992$. ANOVA statistics: $F_{(3,49)}=6.104, p=0.0013$. All combinations were compared using Bonferroni test for multiple comparisons. Vehicle versus CR2Crry: $p<$ 0.001; Vehicle versus CR2fH: $p<0.01$; Vehicle versus CR2CD59: $p=$ 0.45 ; $b$ : Brown-Forsythe test for homogeneity of variance was not significant: $F_{(3,49)}=0.9035 ; p=0.4463$. ANOVA statistics: $F_{(3,49)}=3.505, p=$ 0.0221. All combinations were compared using Bonferroni test for multiple comparisons. Vehicle versus CR2Crry: $p<0.05$; Vehicle versus CR-2fH: $p<0.05$; Vehicle versus CR2CD59: $p=0.5208$; $c$ : BrownForsythe test for homogeneity of variance was not significant: $F_{(3,49)}=$ $1.444 ; p=0.2414$. ANOVA statistics: $F_{(3,49)}=1.636, p=0.1931$. All combinations were compared using Bonferroni test for multiple comparisons. No significant results detected. Figure $2 d$ : repeated-measures (RM) two-way ANOVA was used with Bonferroni test for multiple comparisons. Overall effect comparing the four different treatment groups was computed. ANOVA statistics: $F_{(3,44)}=17.21, p<0.0001$. All combinations were compared using Bonferroni test for multiple comparisons. Vehicle versus CR2Crry: $p<0.001$; Vehicle versus CR2fH: $p<0.001$; CR2Crry or CR2fH versus CR2CD59: $p<0.05$. Figure $2 e$ : one-way ANOVA was used with Bonferroni test for multiple comparisons. Brown-Forsythe test for homogeneity of variance was not significant: $F_{(3,44)}=1.749 ; p=$ 0.1708 . ANOVA statistics: $F_{(3,44)}=19.23, p<0.001$. All combinations were compared using Bonferroni test for multiple comparisons. Figure 2f: RM two-way ANOVA was used with Bonferroni test for multiple comparisons. Overall effect comparing the four different treatment groups was computed. ANOVA statistics: $F_{(3,44)}=19.55, p<0.0001$. All combinations were compared using Bonferroni test for multiple comparisons. Vehicle versus CR2Crry: $p<0.001$; Vehicle versus CR2fH: $p<$ 0.001; CR2Crry and CR-fH versus CR2CD59: $p<0.05$. Figure $2 g$ : RM two-way ANOVA was used with Bonferroni test for multiple comparisons. Overall effect comparing the four different treatment groups was computed. ANOVA statistics: $F_{(3,44)}=35.8, p<0.0001$. All combinations were compared using Bonferroni test for multiple comparisons. Vehicle versus CR2Crry: $p<0.001$; Vehicle versus CR2fH: $p<0.001$; CR2Crry and CR-fH versus CR2CD59: $p<0.05$. Figure $3 d$ : one-way ANOVA was used with Bonferroni test for multiple comparisons. Brown-Forsythe test for homogeneity of variance was significant: $F_{(3,49)}=4.197 ; p=0.01$. ANOVA statistics: $F_{(3,49)}=21.72, p<0.0001$. Due to a significant Brown-Forsythe test, groups were also analyzed using the KW test (non- 


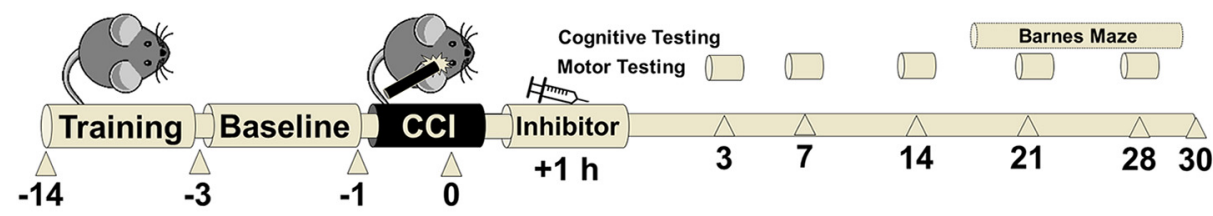

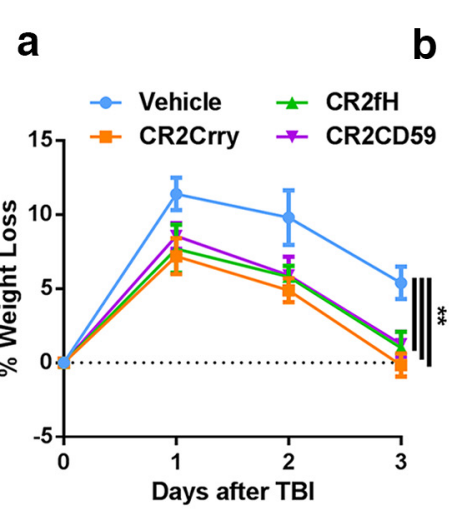

e
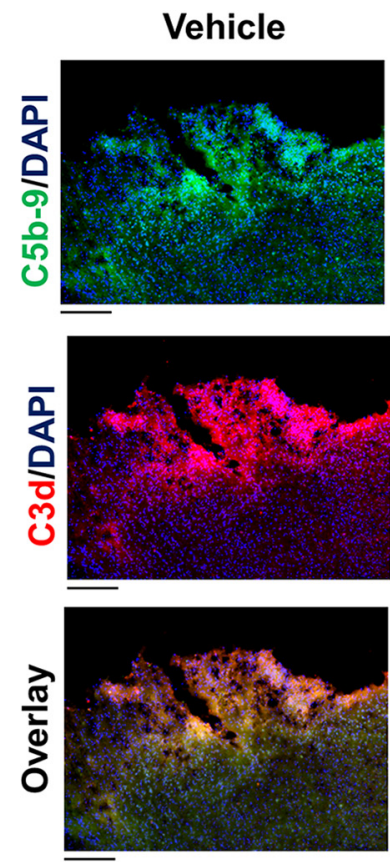

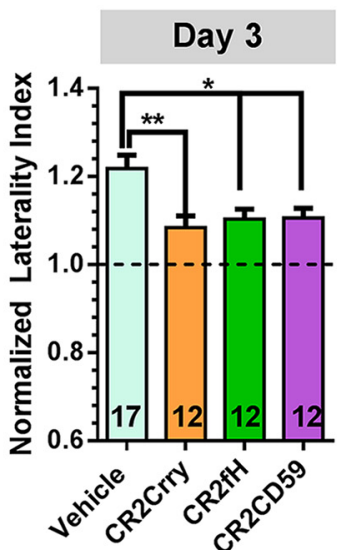

CR2Crry
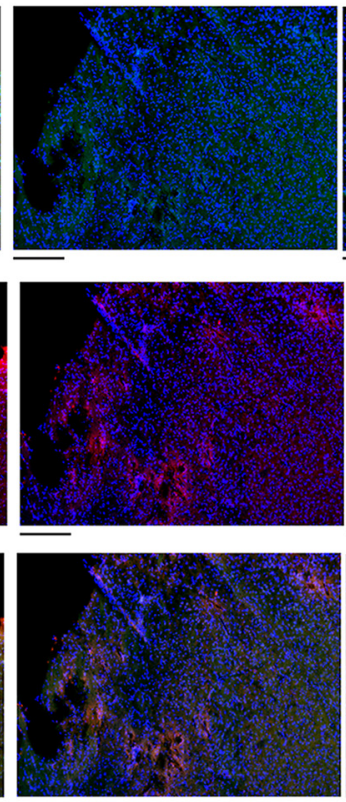

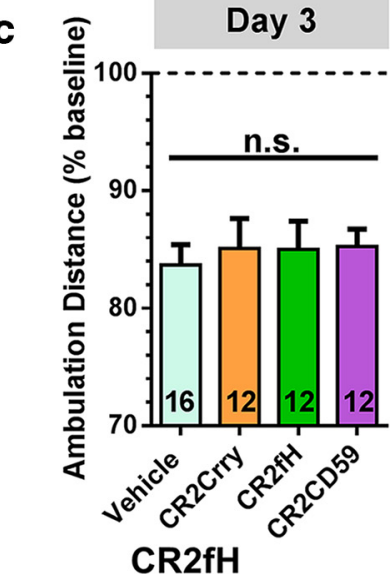

d
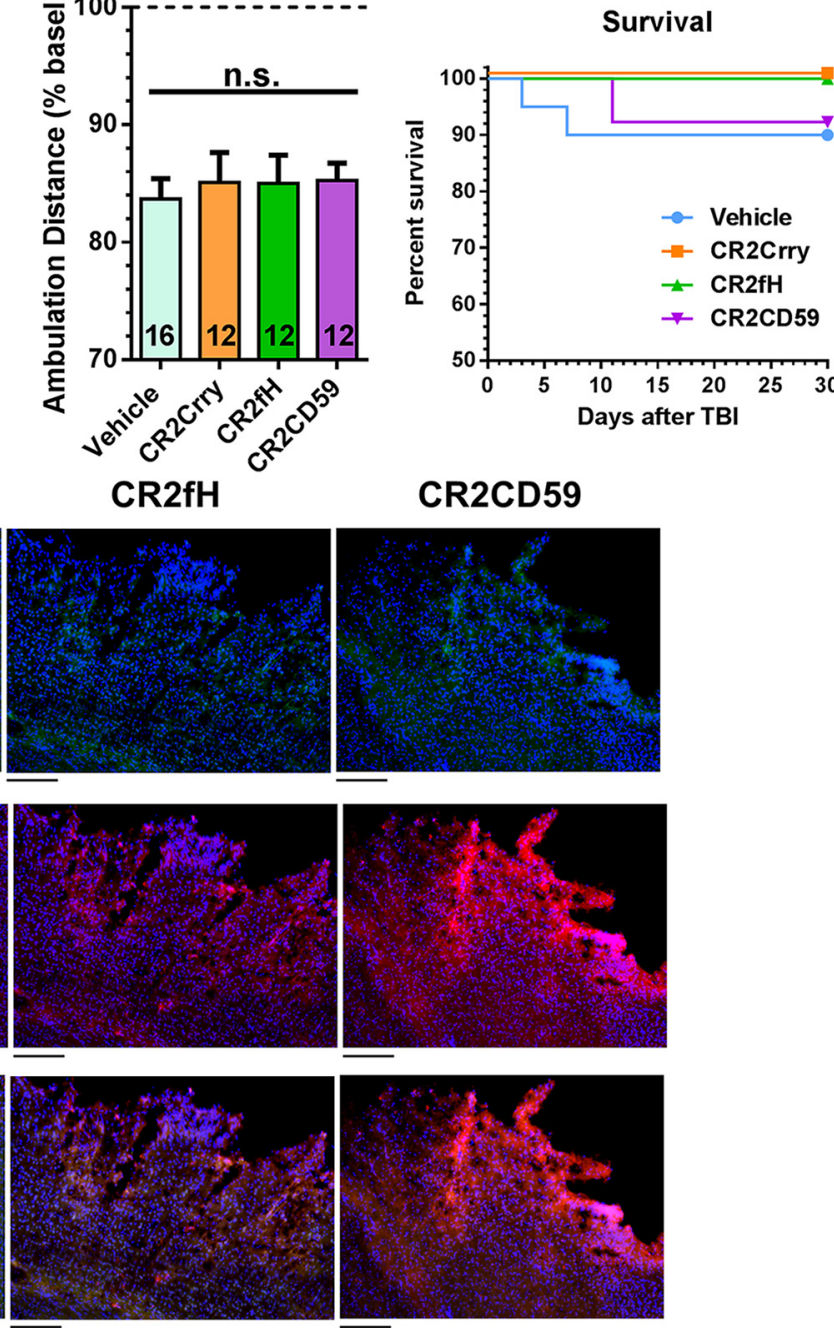

Figure 1. Acute recovery and complement deposition in the brain after $\mathrm{CCl}$ and targeted complement inhibition. $\boldsymbol{a}$, Monitoring of weight loss acutely after CCI showing a significant reduction in weight loss over the $3 \mathrm{~d}$ period in animals treated with CR2Crry, CR2fH, or CR2CD59 compared with vehicle controls. ANOVA for overall effect with Bonferroni's test for multiple comparisons. ${ }^{* *} p<$ 0.01. $n=8$ /group. $\boldsymbol{b}$, Acute assessment of forearm laterality on corner task showing that all three targeted inhibitors result in significant reduction in laterality on day 3 after CCI compared with vehicle controls. One-way ANOVA with Bonferroni's. ${ }^{*} p<0.05,{ }^{* *} p<0.01$. c, Total distance moved by animals in open-field ambulation test showing that targeted complement inhibition does not influence the total distance moved by animals on day 3 compared with vehicle. One-way ANOVA with Bonferroni's. $\boldsymbol{d}$, Kaplan-Meyer survival curve over $30 \mathrm{~d}$ period. Log rank (Mantel-Cox) test was used. No significant difference between groups on pair comparisons. e, IF staining for C5b-9 (MAC, green) and C $3 \mathrm{~d}$ (red) deposition in the perilesional brain $24 \mathrm{~h}$ after CCI. Scale bars, $100 \mu \mathrm{m}$.

parametric) with Dunn's multiple comparisons showing similar results $(\mathrm{KW}=35.56, p<0.0001)$. Figure 3e: one-way ANOVA was used with Bonferroni test for multiple comparisons. Brown-Forsythe test for homogeneity of variance was significant: $F_{(3,49)}=6.683 ; p=0.0007$. ANOVA statistics: $F_{(3,49)}=10.85, p<0.0001$. Due to a significant Brown-Forsythe test, groups were also analyzed using the KW test (nonparametric) with Dunn's multiple comparisons showing similar results (KW $=29.83, p<$ $0.0001)$. Figure $4 a$ : two-way ANOVA was used with Bonferroni test for multiple comparisons. Overall effect comparing the four different treatment groups was computed. ANOVA Statistics: $F_{(3,40)}=11.78, p<$ 0.0001 . All combinations were compared at each day during training and retention using Bonferroni test for multiple comparisons. Figure $4 b$ : two-way ANOVA was used with Bonferroni test for multiple compari- sons. Overall effect comparing the four different treatment groups was computed. ANOVA Statistics: $F_{(3,40)}=18.32, p<0.0001$. All combinations were compared at each day during training and retention using Bonferroni test for multiple comparisons. Figure $4 d$ : one-way ANOVA was used with Bonferroni test for multiple comparisons. Brown-Forsythe test for homogeneity of variance was not significant: $F_{(3,40)}=$ $0.5538 ; p=0.6486$. ANOVA statistics: $F_{(3,40)}=0.5578, p=0.6460$. All combinations were compared using Bonferroni test for multiple comparisons. Figure $5 e$ : one-way ANOVA was used with Bonferroni test for multiple comparisons. Brown-Forsythe test for homogeneity of variance was not significant: $F_{(3,16)}=0.6833 ; p=0.5751$. ANOVA statistics: $F_{(3,16)}=14.15, p<0.0001$. All combinations were compared using Bonferroni test for multiple comparisons. Figure $5 f$ : one-way ANOVA was 
used with Bonferroni test for multiple comparisons. Brown-Forsythe test for homogeneity of variance was not significant: $F_{(3,16)}=0.6833$; $p=0.5751$. ANOVA statistics: $F_{(3,16)}=29.82$, $p<0.0001$. All combinations were compared using Bonferroni test for multiple comparisons. Figure $5 g$ : one-way ANOVA was used with Bonferroni test for multiple comparisons. Brown-Forsythe test for homogeneity of variance was not significant: $F_{(3,16)}=1.767 ; p=$ 0.1940. ANOVA Statistics: $F_{(3,16)}=0.6857, p=$ 0.5738. All combinations were compared using Bonferroni test for multiple comparisons. Figure 5j: one-way ANOVA was used with Bonferroni test for multiple comparisons. Brown-Forsythe test for homogeneity of variance was not significant: $F_{(3,16)}=0.9022 ; p=$ 0.4553. ANOVA statistics: $F_{(3,16)}=17.68, p<$ 0.0001 . All combinations were compared using Bonferroni test for multiple comparisons. Figure $5 k$ : one-way ANOVA was used with Bonferroni test for multiple comparisons. Brown-Forsythe test for homogeneity of variance was not significant: $F_{(3,16)}=0.006 ; p=$ 0.9993. ANOVA statistics: $F_{(3,16)}=8.640, p<$ 0.005 . All combinations were compared using Bonferroni test for multiple comparisons. Figure 6f: one-way ANOVA was used with Bonferroni test for multiple comparisons. Brown-Forsythe test for homogeneity of variance was not significant. ANOVA Statistics: $F=0.043, p=0.9877$. All combinations were compared using Bonferroni test for multiple comparisons. Figure 6g: one-way ANOVA was used with Bonferroni test for multiple comparisons. Brown-Forsythe test for homogeneity of variance was not significant. ANOVA statistics: $F=21.31, p<0.0001$. All combinations were compared using Bonferroni test for multiple comparisons. Figure 6h: one-way ANOVA was used with Bonferroni test for multiple comparisons. Brown-Forsythe test for homogeneity of variance was not significant. ANOVA statistics: $F=9.179, p=0.002$. All combinations were compared using Bonferroni test for multiple comparisons. Figure 6i: one-way ANOVA was used with Bonferroni test for multiple comparisons. Brown-Forsythe test for homogeneity of variance was not significant. ANOVA statistics: $F=8.541, p<0.0026$. All combinations were compared using Bonferroni test for multiple comparisons. Figure 6j: one-way ANOVA was used with Bonferroni test for multiple comparisons. Brown-Forsythe test for homogeneity of variance was not significant. ANOVA statistics: $F=14.44, p=0.0003$. All combinations were compared using Bonferroni test for multiple comparisons. Figure $7 g$ : one-way ANOVA was used with Bonferroni test for multiple comparisons. Brown-Forsythe test for homogeneity of variance was not significant. ANOVA Statistics: $F=6.893, p=0.0102$. All combinations were compared using Bonferroni test for multiple comparisons. Figure 7h: one-way ANOVA was used with Bonferroni test for multiple comparisons. Brown-Forsythe test for homogeneity of variance was not significant. ANOVA statistics: $F=16.29, p=0.0004$. All combinations were compared using Bonferroni test for multiple comparisons. Figure 8a: one-way ANOVA was used with Bonferroni test for multiple comparisons. Brown-Forsythe test for homogeneity of variance was not

\section{Corner Task}

a

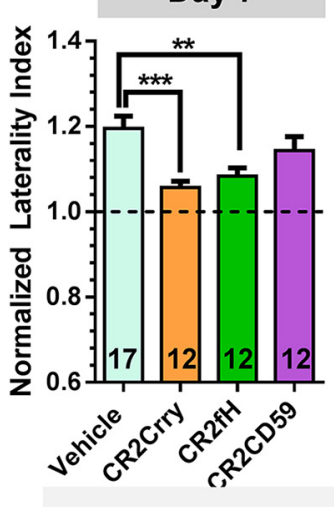

d

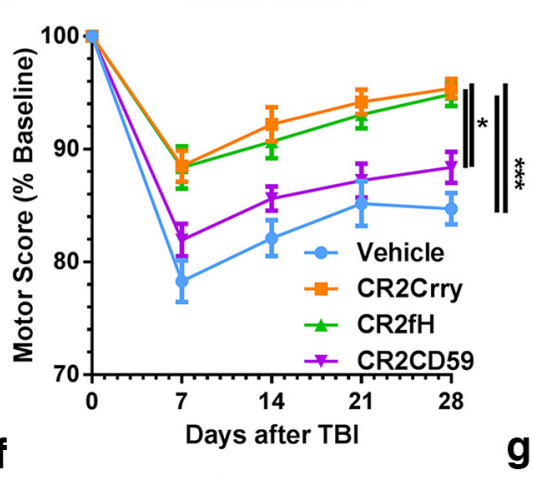

Left Forelimb

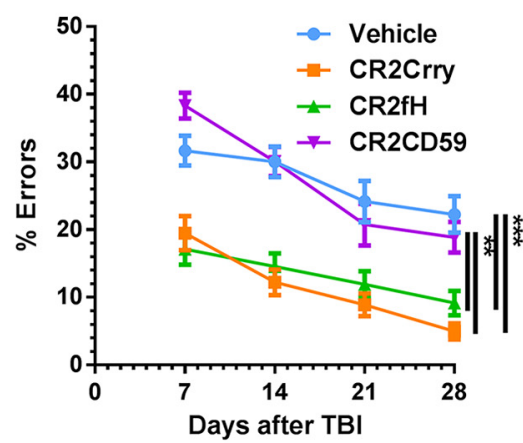

b

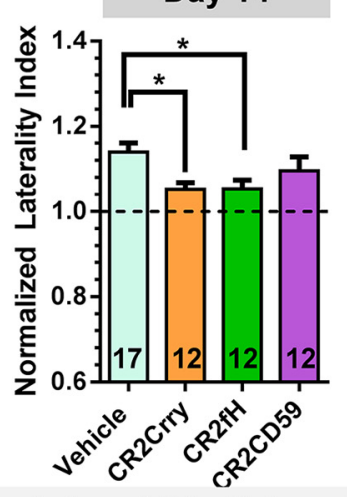

C

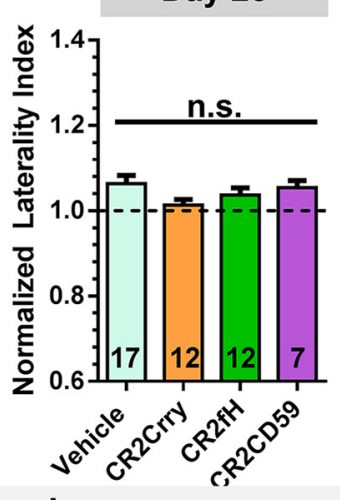

Task

e

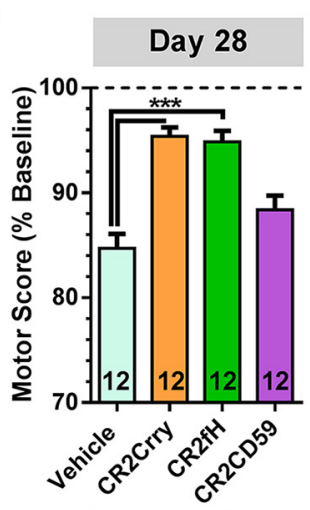

Left Hindlimb

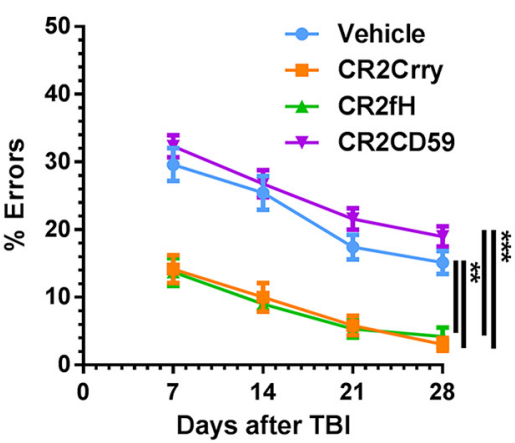

Figure 2. Motor recovery after $\mathrm{CCl}$ and treatment with complement inhibitors. $\boldsymbol{a}-\boldsymbol{c}$, Performance on corner task was assessed weekly after $\mathrm{CCl}$ showing that $\mathrm{CR} 2 \mathrm{fH}$ and $\mathrm{CR} 2 \mathrm{Crry}$, but not $\mathrm{CR} 2 \mathrm{CD} 59$, resulted in significantly faster recovery of forearm laterality compared with vehicle. One-way ANOVA with Bonferroni's multiple comparisons. ${ }^{*} p<0.05$, ${ }^{* *} p<0.01$, ${ }^{* * *} p<0.001$. d, Motor scores on ladder task were assessed weekly after CCl showing that CR2fH and CR2Crry, but not CR2CD59, resulted in significantly high motor scores throughout $28 \mathrm{~d}$ of recovery compared with both vehicle controls and animals treated with CR2CD59. RM two-way ANOVA with Bonferroni's test for multiple comparisons. ${ }^{*} p<0.05,{ }^{* *} p<0.01,{ }^{* * *} p<0.001$. Error bars $=$ mean \pm SEM. e, Motor scores on day 28 after CCl showing significantly higher scores in CR2Crry and CR2fH-treated mice, but not CR2CD59treated mice compared with vehicle controls. One-way ANOVA with Bonferroni's multiple comparisons. ${ }^{*} p<0.05,{ }^{* *} p<0.01$, ${ }^{* * *} p<0.001 . \boldsymbol{f}, \boldsymbol{g}$, Forelimb and hindlimb errors showing a significant reduction in the number of errors after CR2Crry or CR2fH therapy compared with both CR2CD59 and vehicle. RM two-way ANOVA with Bonferroni's test for multiple comparisons. ${ }^{*} p<$ $0.05,{ }^{* *} p<0.01,{ }^{* * *} p<0.001$. Error bars $=$ mean \pm SEM.

significant: $F_{(2,18)}=0.6302 ; p=0.5438$. ANOVA statistics: $F_{(2,18)}=$ $10.59, p<0.0001$. All combinations were compared using Bonferroni test for multiple comparisons. Figure $8 b$ : one-way ANOVA was used with Bonferroni test for multiple comparisons. Brown-Forsythe test for homogeneity of variance was not significant: $F_{(2,18)}=2.895 ; p=0.0812$. ANOVA statistics: $F_{(2,18)}=10.57, p<0.0001$. All combinations were compared using Bonferroni test for multiple comparisons. Figure $8 c$ : 

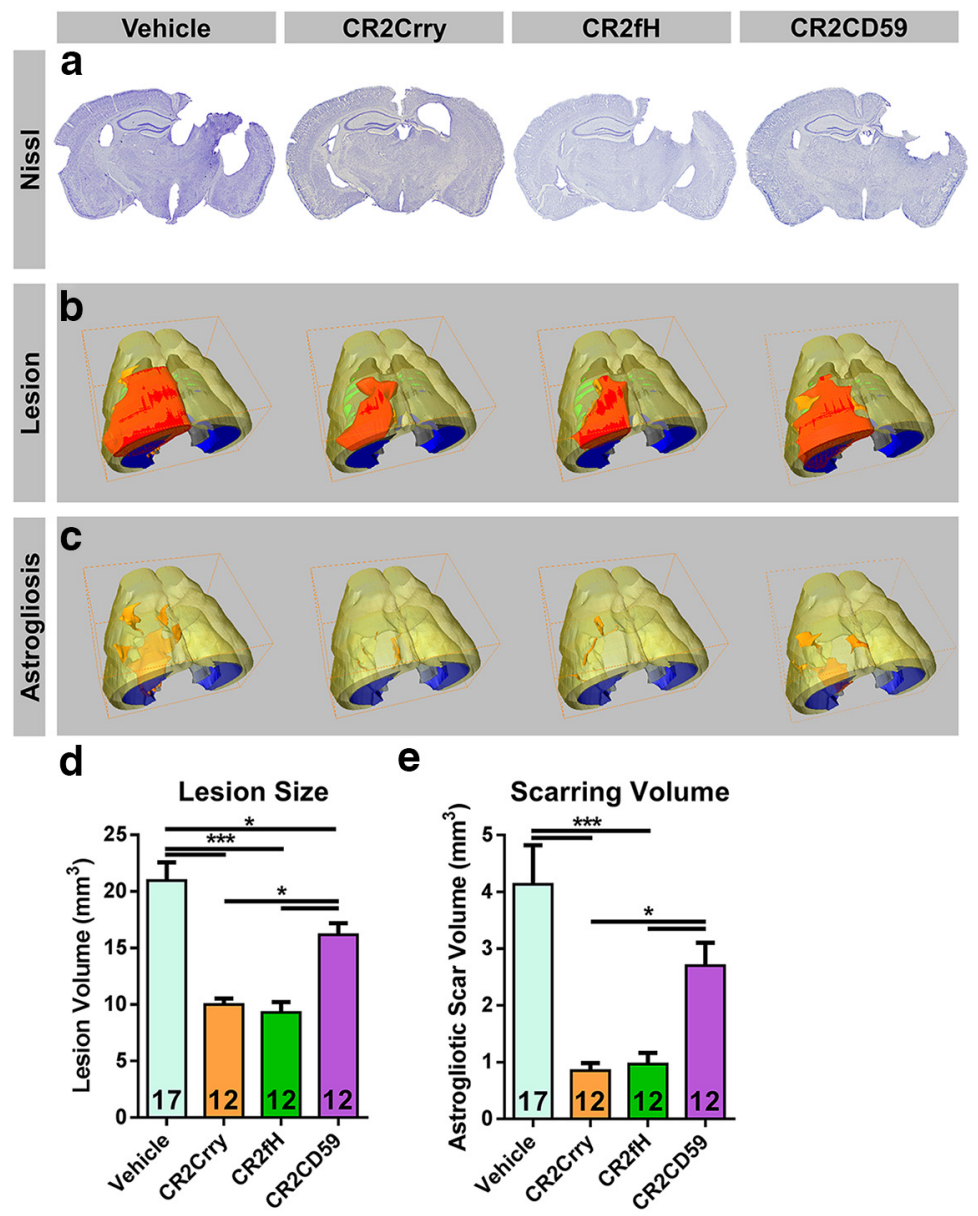

e

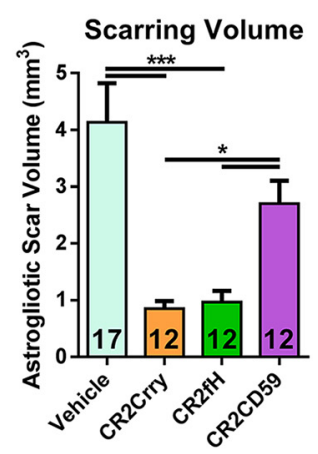

Figure 3. Effect of targeted complement inhibition on lesion volume and scarring after $\mathrm{CCl}$. $\boldsymbol{a}$, Representative Niss stained brain section from the different treatment groups. $\boldsymbol{b}, 3 \mathrm{D}$-reconstructed brains showing lesion (red) and scarring (orange) $30 \mathrm{~d}$ after TBI. Brains were reconstructed from Nissl quantification as described in Materials and Methods, Lesion volume reconstruction. Yellow, Cortex; blue, hippocampus; green, basal ganglia; gray, white matter. $\boldsymbol{c}$, Similar to $\boldsymbol{b}$ but showing only scarring in the same brains shown in $\boldsymbol{b}$. $\boldsymbol{d}, \boldsymbol{e}$, Quantification of lesion volume and scarring at $30 \mathrm{~d}$ after $\mathrm{CCl}$ showing significant reduction in lesion volume across the different treatment groups compared with vehicle controls. However, CR2fH and CR2Crry resulted in more effective reduction in overall lesion compared with CR2CD59. d, CR2Crry and CR2fH were effective at reduction in scarring $30 \mathrm{~d}$ after TBI, but not CR2CD59. One-way ANOVA with Bonferroni's multiple comparisons. $n=12-17 /$ group. ${ }^{*} p<0.05,{ }^{* *} p<0.001$. Error bars $=$ mean \pm SEM.

one-way ANOVA was used with Bonferroni test for multiple comparisons. Brown-Forsythe test for homogeneity of variance was not significant: $F_{(2,18)}=0.3455 ; p=0.7125$. ANOVA statistics: $F_{(2,18)}=6.41, p<$ 0.001 . All combinations were compared using Bonferroni test for multiple comparisons. Figure 8d: two-way ANOVA was used with Bonferroni test for multiple comparisons. Overall effect comparing treatment groups to controls was computed. ANOVA statistics: $F_{(2,18)}=18.91, p<0.0001$. All combinations were compared at each day during training and retention using Bonferroni test for multiple comparisons.

Recombinant proteins. Recombinant complement inhibitor CR2fH used in this study was constructed, expressed, and purified as previously described (Huang et al., 2008). All proteins used in these experiments were subjected to quality control for complement inhibitory activity using chicken red blood cell lysis assay and zymosan assay as described previously (Huang et al., 2008).

Animal surgeries and treatment. Adult male C57BL/6 were obtained from The Jackson Laboratory and allowed 1 week of acclimation before use. Adult animals were 12 weeks old at the time of CCI surgeries that were performed as previously described (Smith et al., 1995; Fox et al., 1998). Following anesthesia using ketamine $(80-100 \mathrm{mg} / \mathrm{kg}$, i.p.) and xylazine (10 mg/kg, i.p.), the mouse head was shaved, prepped with Betadine, and fixed on a stereotactic frame using (Kopf Instruments). A longitudinal midline incision was performed to expose the skull. Using a cotton applicator, the surface of the skull was cleaned and left to dry for $1 \mathrm{~min}$. Using a drill (RWD Life Sciences), a hole was drilled halfway between lambda and bregma with a diameter of $4 \mathrm{~mm}$ situated $0.5 \mathrm{~mm}$ lateral to midline. A thin layer of the bone was kept on top of dura to prevent cutting through the brain, then using a forceps, the bone was cleared from the top of the brain exposing the dura. Contusions were made with a pneumatic impactor device (Infinite Horizon, Precision Scientific). The impactor parameters were set to tip size of 3 $\mathrm{mm}$, depth of $2.5 \mathrm{~mm}$ (moderate CCI), velocity of $5.25 \mathrm{~m} / \mathrm{s}$, dwell time of $100 \mathrm{~ms}$, and a $10^{\circ}$ angle. The impact was then delivered to the brain on intact dura. Sham operated mice underwent a craniotomy only. Animals with bone hits were excluded. Wounds were sutured and, upon recovery from anesthesia, animals were be placed in their home cages and given ad libitum access to soft food and water. All surgical procedures were performed under aseptic conditions, and body temperature was monitored by a rectal probe and maintained at $37 \pm 0.5^{\circ} \mathrm{C}$ with a heating pad.

At $1 \mathrm{~h}$ after CCI, vehicle (PBS), CR2Crry (10 $\mathrm{mg} / \mathrm{kg})$, CR2fH (16 mg/kg), or CR2CD59 (6 $\mathrm{mg} / \mathrm{kg}$ ) were administered intravenously as a single dose via the tail vein. CR2Crry and CR2fH treatments were also administered $12 \mathrm{~h}$ after CCI. Doses were determined based on prior dose-response experiments that determined minimum dose required for providing maximum protection against ischemia reperfusion injury (Atkinson et al., 2005; Huang et al., 2008; Marshall et al., 2014). The same studies determined that the circulatory half-life of each protein was similar and in the range of $6.6-8.8$ h. Animals were killed at $30 \mathrm{~d}$ after TBI for histological analyses unless a humane endpoint determined by loss of $>15 \%$ of total body weight over $48 \mathrm{~h}$ is reached, at which time animals would be killed and therefore excluded from histological analyses at $30 \mathrm{~d}$. There were no procedure-related mortalities, and three animals ( 2 from vehicle group and 1 from CR2CD59 group) were killed before $30 \mathrm{~d}$ for reaching the humane endpoint.

Lesion volume reconstruction. Lesion size including scarring was estimated using Nissl staining of serial brain sections. Brains were harvested at $30 \mathrm{~d}$ after CCI following cardiac perfusion with ice-cold PBS and 4\% paraformaldehyde (PFA). Brains were then cryoprotected using 30\% sucrose in PFA and then cut into $40 \mu \mathrm{m}$ thick coronal sections. Serial sections $200 \mu \mathrm{m}$ apart were mounted and stained using cresyl violet as previously described (Türeyen et al., 2004). Slides were then imaged using Olympus BX61 light microscope with Visiopharm image acquisition and analysis software. To quantify and anatomically locate lesions, Nissl-stained images were aligned to Paxinos' brain atlas, and lesion location (infarct and astrogliosis) was mapped to the atlas sections. Following lesion mapping, 3D reconstruction of lesions and brains was conducted on Amira 6.2 (FEI), and volume was computed in cubic millimeters.

Assessment of motor recovery. Forelimb asymmetry was measured using corner test as described previously (Zhang et al., 2002). Laterality was measured at baseline and after surgery. Normalized laterality index was calculated as $[($ laterality at time $t)+2] /[($ baseline laterality $)+2)]$. Openfield locomotor testing was performed as described previously (Alawieh et al., 2015b). Animals were videotaped and videos were analyzed using Autotyping toolbox (University of Pennsylvania). Total distance moved 
and the duration of movement were computed to assess ambulation, and time spent at the center versus periphery was used to assess differences in anxiety levels. The ladder walk task was performed as previously described (Metz and Whishaw, 2009). Animals were tested for three trials per session and a total of 40 steps are scored per animal per trial. Each paw placement was scored on a scale from 0 to 6 as follows: 0 , total miss; 1 , deep slip; 2, slight slip; 3, replacement; 4, correction; 5, partial placement; 6 , correct placement. The average score of all steps and the number of errors are recorded as the ladder motor score per trial. The average of three trials is used for each animal. The ladder task was performed at baseline and at designated times after CCI.

Assessment of cognitive recovery. Barnes maze was used to assess spatial learning after TBI using a previously described protocol (Patil et al., 2009). Animals were trained for two trials per day for $5 \mathrm{~d}$ starting at day 23 after CCI while being video-recorded. Videos were then automatically analyzed using Autotyping toolbox (University of Pennsylvania) to compute the total path length and the number of error pokes before animals reach the escape box.

Immunohistochemical staining. For immunohistochemical (IHC) staining, brains were harvested after mice were intracardially perfused with PBS followed by $4 \%$ paraformaldehyde, fixed overnight, and then cryoprotected in $30 \%$ sucrose. Brains were then cut into $40 \mu \mathrm{m}$ sections using a cryostat, and sections between 0 and $-2 \mathrm{~mm}$ relative to bregma were used for staining. Free-floating sections were incubated with $3 \% \mathrm{H}_{2} \mathrm{O}_{2}$ followed by $0.1 \%$ Triton-X in PBS, blocked in 5\% donkey serum, and incubated with primary antibodies overnight. Sections were then washed, incubated with HRP-conjugated secondary antibodies, and developed using DAB (ImmPress DAB, Vector Laboratories). Sections were mounted on slides, counterstained with hematoxylin, and imaged using an Olympus BX61 light microscope with Visiopharm image acquisition and analysis software (Visiopharm). Antibodies used in IHC studies were antiC3d (R\&D Systems, AF2655; 1:50), anti-IgM (Sigma-Aldrich, A8786; 1:200), anti-Ibal (Abcam, ab5076; 1:200), and anti-PSD95 (Abcam, ab18258; 1:100) with HRP-conjugated anti-rabbit or anti-goat secondary (Vector Laboratories).

Immunofluorescence staining and imaging. A similar approach was used to stain $40 \mu \mathrm{m}$ sections as outlined above. Primary antibodies used include anti-C3d (R\&D Systems, catalog \#AF2655; 1:50), anti-Iba1 (Abcam, catalog \#ab5076; 1:200), anti-Mac2 (R\&D Systems, catalog \#AF1197; 1:300-1:400), anti-GFAP (Invitrogen, catalog \#13-0300; 1:200), anti-C5b-9 (Calbiochem, catalog\#204903; 1:100), anti-MAP2 (Abcam, catalog\#ab32454; 1:200), antiDcx (Abcam, catalog \#ab18723; 1:200), anti-BrdU (Abcam, catalog\#ab6326; 1:100), and anti-NeuN (Millipore, catalog \#MAB377; 1:200). Secondary antibodies used were as follows: donkey anti-goat AlexaFluor 488 and $555 \mathrm{~nm}$; donkey anti-rabbit AlexaFluor 488, 555, and $647 \mathrm{~nm}$; and donkey anti-rat AlexaFluor 488 and $555 \mathrm{~nm}$. DAPI (Vector Laboratories) was used as nuclear stain. Slides were mounted with Vectashield mounting media ( \pm DAPI) before microscopy. Epifluorescence microscopy was used to image full brain sections using an Olympus BX61 light microscope with Visiopharm image acquisition and analysis software. Intensity-based analysis was automatically computed by using MATLAB (MathWorks) by normalizing the intensity of signal to the area of tissue displayed per random filed. Super-resolution imaging was performed using a Zeiss LSM 880 confocal microscope, and Airy Scan was used for high-resolution fields. Fields were imaged using $40 \times$ water and $63 \times$ oil objectives. Sequential imaging was performed for multiple channels, and a stack covering $30 \mu \mathrm{m}$ thickness was acquired at $1 \mu \mathrm{m}$ resolution. 3Dreconstructed images of the full stack or 2D slices of the maximal intensity focal point were shown. Image analysis was performed using both ZEN (Zeiss) and ImageJ (NIH).

\section{Results}

C3 activation via the ACP exacerbates chronic deficits after $\mathrm{CCI}$ in a MAC-independent mechanism

We first investigated the effect of site-targeted inhibition of all complement pathways (CR2Crry), the alternative pathway (CR2fH), or the MAC (CR2CD59), administered $1 \mathrm{~h}$ after CCI, on general health (weight) and motor function over $28 \mathrm{~d}$ postinjury (Fig. 1).
The three interventions resulted in a significant reduction in acute weight loss up to $3 \mathrm{~d}$ after TBI compared with vehicle controls (Fig. 1a), and resulted in significant reduction in forearm laterality on corner task (Fig. 1b). Although, there was not a significant difference between the three inhibitors, the protective effects of CR2Crry were quantitatively higher than CR2fH or CR2CD59 on both measures (Fig. 1a,b). As anticipated from a moderately severe CCI, animals did not develop severe motor disability, and the total ambulation distance was not significantly increased by complement inhibition compared with vehicle controls acutely after TBI (Fig. 1c). Similarly, targeted complement inhibition did not affect survival after CCI during the $28 \mathrm{~d}$ of recovery period, although $\mathrm{CR} 2 \mathrm{fH}$ and CR2Crry-treated animals trended toward lower $28 \mathrm{~d}$ mortality compared with both CR2CD59 and vehicle controls (Fig. 1d). We then used immunofluorescence staining to assess the efficacy of the different constructs in inhibiting complement in vivo (Fig. 1e). All three inhibitors reduced the deposition of the terminal MAC (C5b-9) in the perilesional brain compared with vehicle controls. However, unlike CR2Crry and CR2fH, CR2CD59 did not reduce the deposition of C3 activation products (C3d) $24 \mathrm{~h}$ after CCI and treatment (Fig. 1e). These inhibitors therefore allow us to dissect the role of early versus terminal complement activation products, and whether their selective inhibition is sufficient to maintain neuroprotection after CCI.

We next assessed whether the neuroprotective effects of complement inhibition by each inhibitor persists over $28 \mathrm{~d}$ of recovery. Despite significant recovery, animals treated with CR2fH or CR2Crry showed a sustained reduction in forearm laterality on corner task up to $14 \mathrm{~d}$ after injury. In contrast, animals treated with CR2CD59 were not significantly different from controls starting $7 \mathrm{~d}$ after TBI (Fig. 2a-c). There was no significant difference in forearm laterality at $28 \mathrm{~d}$ after TBI across all groups due to the significant recovery of the vehicle-treated animals and the lack of sensitivity of the corner task at later time points. We next used a more sensitive assessment of motor recovery, namely the ladder walking task, to assess coordinate forelimb and hindlimb use and paw placement after TBI (Fig. $2 d-g$ ). Control animals showed a robust reduction in overall motor scores defined as described previously (Metz and Whishaw, 2009) and an increase in the number of errors by ipsilateral limbs, which persisted over $28 \mathrm{~d}$ of assessment (Fig. 2d-g). Treatment with either CR2Crry or CR2fH significantly increased motor scores resulting in a more prominent and faster recovery compared with vehicle control, an effect that was significant even $28 \mathrm{~d}$ after initial injury and treatment (Fig. 2d,e). In contrast, CR2CD59 did not significantly improve recovery in motor scores compared with vehicle controls between 7 and $28 \mathrm{~d}$ after injury, and the overall recovery in motor scores in CR2CD59-treated mice was significantly lower compared with CR2Crry or CR2fH (Fig. 2d). We also assessed the number of errors (or limb faults), and demonstrated that both CR2Crry and $\mathrm{CR} 2 \mathrm{fH}$ treatment resulted in a significant reduction in forelimb and hindlimb errors compared with vehicle and CR2CD59treated animals (Fig. 2f,g). This indicates that although inhibition of the MAC by CR2CD59 facilitates acute motor recovery after TBI similar to C3 inhibition, this protection is not sustained chronically, except when C3 cleavage is inhibited by CR2Crry or CR2fH.

\section{C3 activation promotes secondary lesion expansion and scarring after CCI predominantly via the ACP}

Previous studies have shown that inhibition of C3 or MAC result in significant acute reduction in acute injury after TBI (Leinhase 


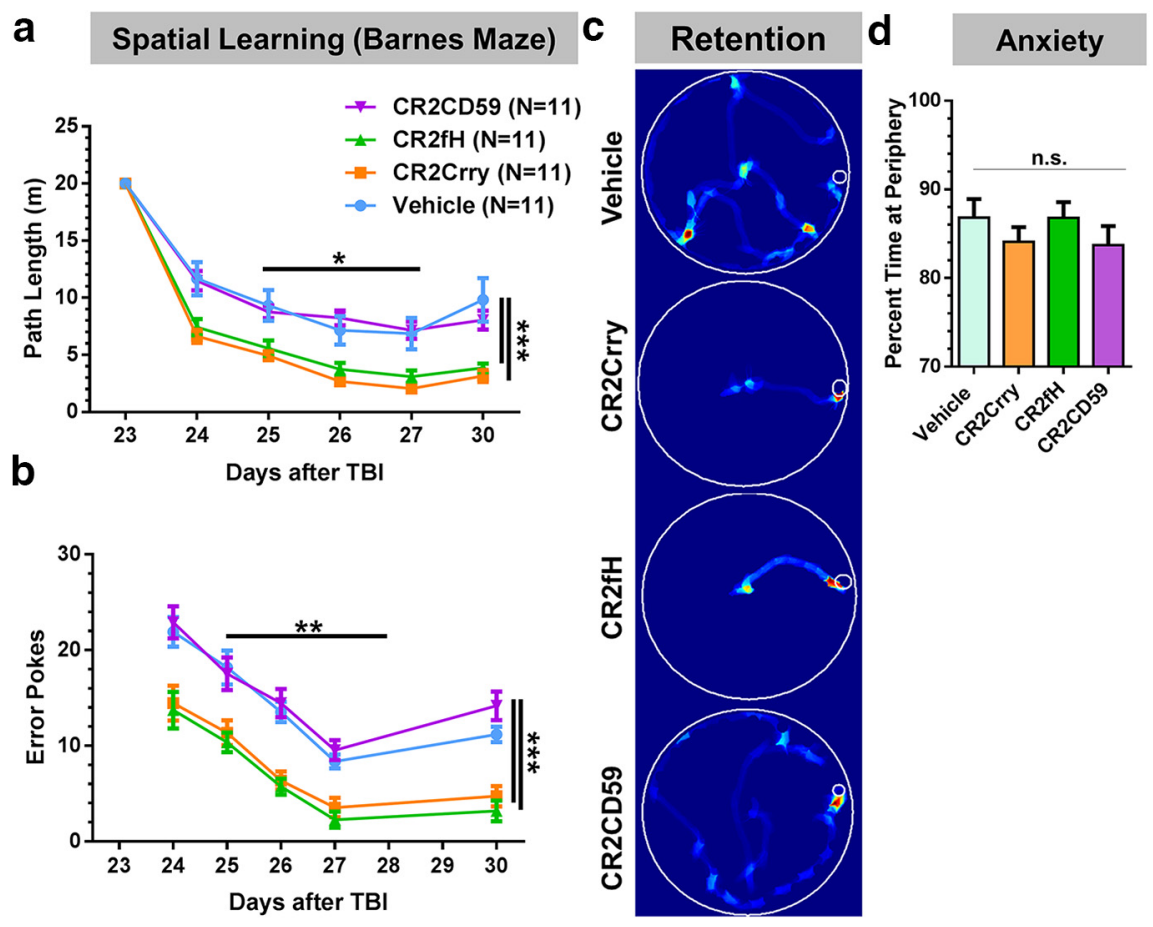

Figure 4. Targeted inhibition of C3-cleavage significantly improves cognitive recovery after CCl. $\boldsymbol{a}$-c, Spatial learning and memory were assessed using the Barnes maze task. $\boldsymbol{a}$, Path length traveled by the animal before reaching the escape hole and (b) the number of error pokes each animal made before reaching the scape hole. Treatment with CR2Crry and CR2fH but not CR2CD59 resulted in significant improvement compared with vehicle controls. RM two-way ANOVA with Bonferroni's multiple comparisons. $n=7-12$ /group. ${ }^{*} p<0.05,{ }^{* *} p<0.01,{ }^{* * *} p<0.001$. $\boldsymbol{d}$, Anxiety level was assessed using the percentage time spent at the periphery of an open field. Animals among the different groups did not show significant differences in anxiety levels. One-way ANOVA with Bonferroni's multiple comparisons. $n=7-12 /$ group. Data shown as mean \pm SEM.

et al., 2006a,b, 2007; Stahel et al., 2009; Ruseva et al., 2015; Rich et al., 2016); therefore, we assessed whether acute inhibition of different complement pathways can differentially affect secondary lesion expansion and astrogliosis that occurs chronically after TBI. Using 3D reconstruction of serial brain sections stained by Nissl, both lesion volume and the extent of tissue scarring were assessed (Fig. 3). Targeted inhibition of the MAC significantly reduced lesion volume but did not significantly inhibit astrogliotic scarring $30 \mathrm{~d}$ after CCI (Fig. 3a-e). However, inhibition of C3 convertase by CR2Crry or inhibition of the ACP by CR2fH significantly reduced lesion volume compared with controls, more effectively than CR2CD59 (Fig. 3d). Both CR2Crry and CR2fH also resulted in significant reduction in astrogliotic scarring at $30 \mathrm{~d}$ after injury compared with controls (Fig. 3e). These data show that inhibition of the MAC may be sufficient to prevent tissue loss after TBI, but this effect is less robust compared with inhibition of C3convertase activity that provides optimal histopathological protection.

\section{Targeted inhibition of $\mathrm{C} 3$ activation but not of MAC formation improves cognitive recovery after CCI}

TBI is associated with significant cognitive impairments that are often more prominent than motor deficits in both human patients and animal models (Fox et al., 1998; Arciniegas et al., 2002). Therefore, we assessed the effect of complement inhibition on spatial learning and retention of spatial memory acquired 24-27 $\mathrm{d}$ after CCI using the Barnes maze. Compared with vehicle controls, only animals treated acutely with CR2fH and CR2Crry showed significantly faster learning curves and a significantly lower number of errors during both acquisition and retention of spatial memory (Fig. 4a-c). Animals treated with CR2CD59 were indis- tinguishable from controls during both acquisition and retention phases. To control for potential differences in anxiety levels in the different groups before the task, animals were assessed for time spent at the periphery versus center of an open field as a surrogate marker of anxiety levels. No differences were seen between the different groups (Fig. $4 d)$. Therefore, inhibition of C3 acutely is required to facilitate and potentiate cognitive recovery whereas acute MAC deposition does not relate to delayed cognitive recovery after TBI.

Targeted inhibition of C3 activation but not of MAC formation inhibits chronic neuroinflammation after CCI

The complement system is responsible for initiation and amplification of neuroinflammation in stressed and injured CNS. Therefore, we assessed the effect of inhibiting different complement pathways on chronic neuroinflammation after CCI by immunostaining for a panel of neuroinflammatory markers. Inflammation in the CNS is associated with deposition of IgM and $\mathrm{C} 3$ breakdown products (C3d opsonins) that tag injured and stressed cells and mediate activation of immune cells in the brain. Acute treatment with CR2Crry and $\mathrm{CR} 2 \mathrm{fH}$ significantly reduced both $\operatorname{IgM}$ and $\mathrm{C} 3 \mathrm{~d}$ deposition in the perilesional cortex $30 \mathrm{~d}$ after TBI indicating suppression of ongoing neuroinflammation (Fig. 5a,b). Treatment with CR2CD59 was not associated with as significant reduction in these markers (Fig. 5a,b). Deposition of IgM and C3d in vehicle and CR2CD59-treated animals was associated with accumulation of Iba1 + microglia/macrophages in the perilesional cortex that exhibited predominantly an amoeboid rather than branched morphology consistent with a proinflammatory phenotype (Fig. 5c,e,f). CR2Crry and CR2fH treatment were associated with significant inhibition in the number of Iba1 + cells as well as a lower percentage of those cells exhibiting an amoeboid morphology (Fig. $5 c, e, f$ ). To assess whether there is a continued deposition of the MAC in the perilesional brain $30 \mathrm{~d}$ after TBI similar to C3d, we used immunofluorescent (IF) staining for the C5b-9 complex and show a less prominent MAC deposition compared with C3d deposition that was not significantly different across the different treatment groups (Fig. $5 d, g$ ).

Activation of microglia and ongoing astrocytic expansion are markers of continuous noxious insults to the brain, and in vehicletreated animals, we show significant and robust microgliosis and astrogliosis with continuous infiltration of proliferating (Mac2+) microglia/macrophages and GFAP + astrocytes into the perilesional cortex, basal ganglia and hippocampus (Fig. $5 h-k$ ), which often extended to the opposite hemisphere. A similar response to vehicle was also observed in CR2CD59-treated mice indicating that inhibiting the MAC is not sufficient to limit the propagation of neuroinflammation chronically after TBI. However, inhibition of all pathways or the ACP by CR2Crry or CR2fH, respectively, significantly reduced the number of Mac2 + and GFAP + cells in the perilesional brain indicating a faster resolution of inflammation in these animals (Fig. 5h-k). 

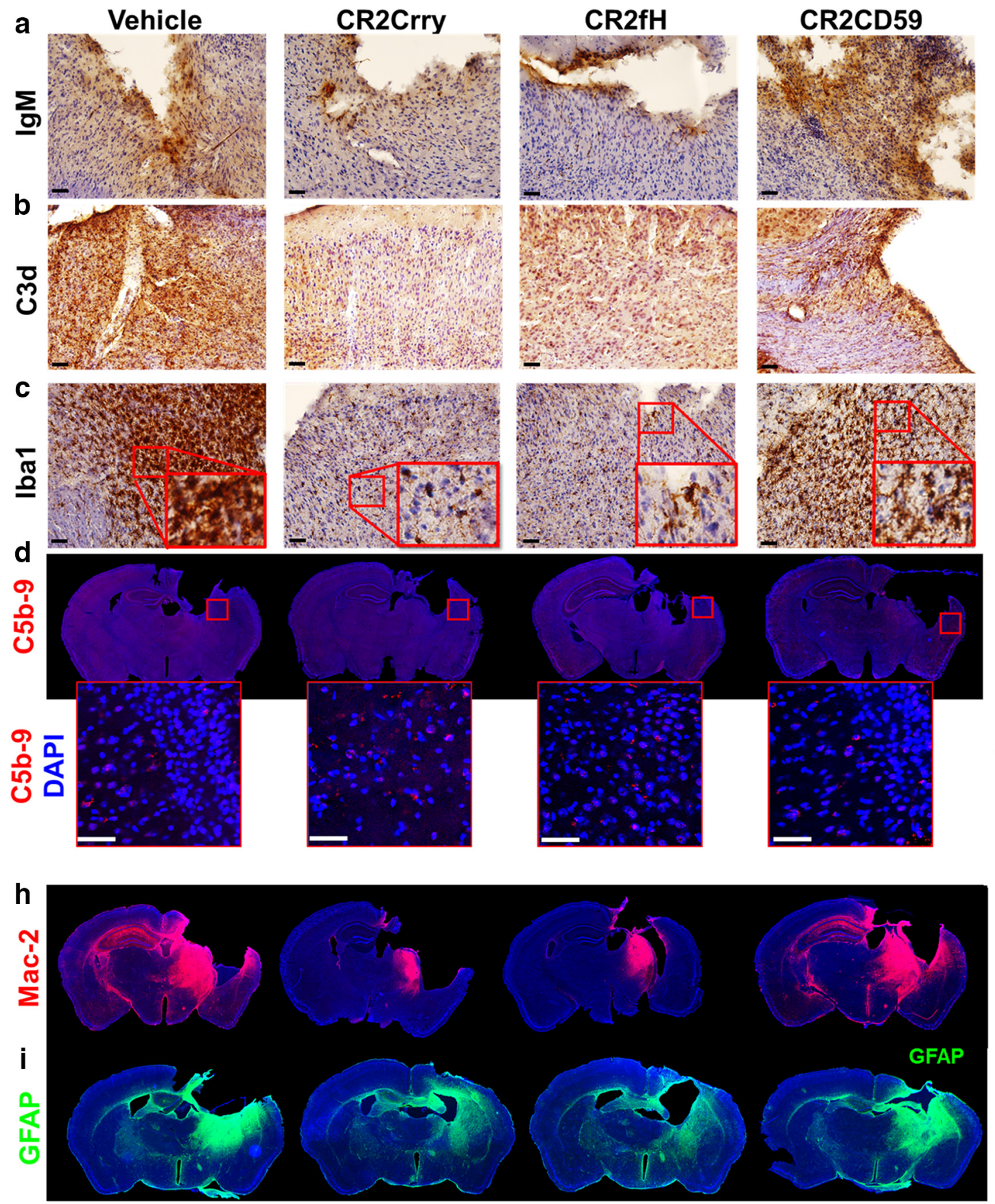
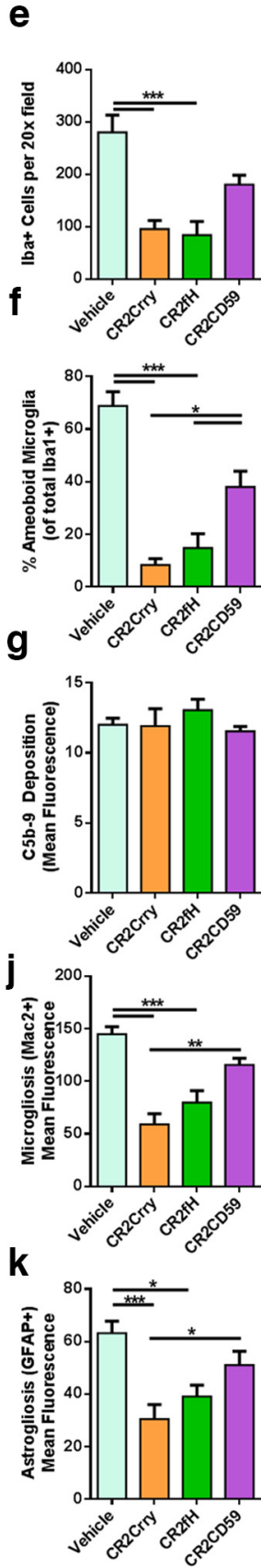

Figure 5. Targeted inhibition of $\mathrm{C} 3$ activation limits chronic neuroinflammation after C(I. $\boldsymbol{a}-\boldsymbol{c}$, Representative IHC staining of IgM deposition (a), C3d deposition (b), and Iba $1+$ cells (c) in the perilesional brain $30 \mathrm{~d}$ after $\mathrm{CCl}$. Scale bars, $50 \mu \mathrm{m}$. $\boldsymbol{d}$, Representative IF images of brain sections stained for (5b-9 (MAC) showing minimal MAC deposition $30 \mathrm{~d}$ after TBI. Red squares, High-magnification confocal microscopy of perilesional brain regions stained for $(5 \mathrm{~b}-9$. Scale bars, $50 \mu \mathrm{m}$. $\boldsymbol{e}, \boldsymbol{f}$, Quantification of number and morphology of Iba $1+$ cells showing significantly lower number of Iba $1+$ cells and percentage of amoeboid Iba $1+$ cells in CR2Crry and CR2fH-treated animals. One-way ANOVA with Bonferroni's multiple comparisons. $n=5 /$ group. ${ }^{*} p<0.05$, ${ }^{* * *} p<$ 0.001. $\boldsymbol{g}$, Quantification of (5b-9 deposition in the perilesional brain from the high fields shown in $\boldsymbol{d}$ demonstrating no significant difference in mean intensity of $(5 \mathrm{~b}-9$ between vehicle-treated animals and the other treatment groups. One-way ANOVA with Bonferroni's multiple comparisons. $n=5 /$ group. $p=0.573$. $\boldsymbol{h}, \boldsymbol{i}$, Representative full-brain IF staining for Mac2 and GFAP $30 \mathrm{~d}$ after CCl. $\boldsymbol{j}, \boldsymbol{k}$, Quantification of $\boldsymbol{f}$ and $\boldsymbol{g}$ showing significant reduction in Mac2 + cells and GFAP + cells in animals treated with CR2Crry or CR2fH but not CR2CD59. One-way AN0VA with Bonferroni's multiple comparisons. $n=7-9 /$ group. ${ }^{*} p<0.05,{ }^{* *} p<0.01,{ }^{* * *} p<0.001$. All error bars represent mean \pm SEM.

Inhibiting $\mathrm{C} 3$ activation increases neuroblast migration and does not interfere with SVZ neurogenesis

Previous work has implicated a role for C3 in neurogenesis and neuronal migration after brain injury (Rahpeymai et al., 2006). We have also shown that maintaining a low level of complement activation in the brain after ischemic stroke results in increased neurogenesis and neuronal migration compared with comple- ment deficiency (Alawieh et al., 2015b). Therefore, we assessed whether inhibition of complement using CR2Crry or CR2fH negatively impacts neurogenesis and neuronal migration after TBI. We used intraperitoneal BrdU injections administered every other day starting on day 3 after CCI for birth-dating newly formed neurons, and brain sections were then stained for doublecortin (Dcx) and BrdU to assess both neurogenesis and migration of 
a

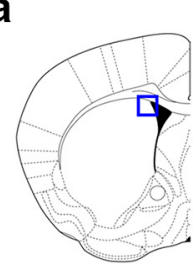

b

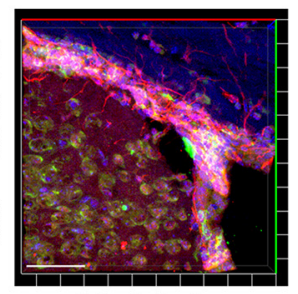

Vehicle

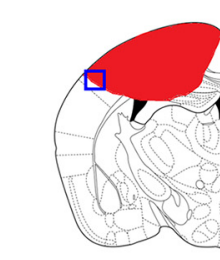

C
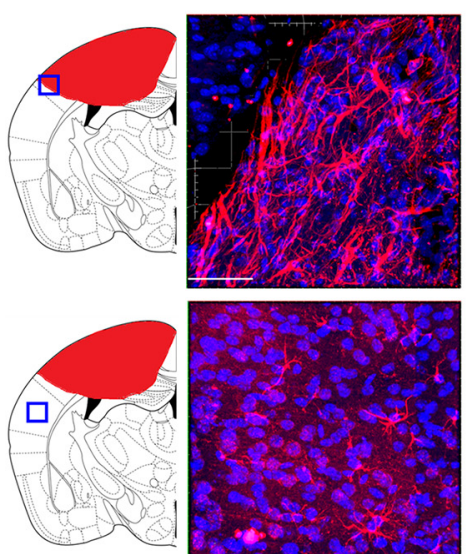

d

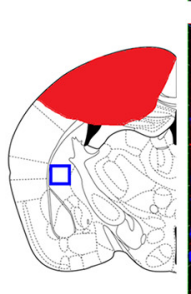

e

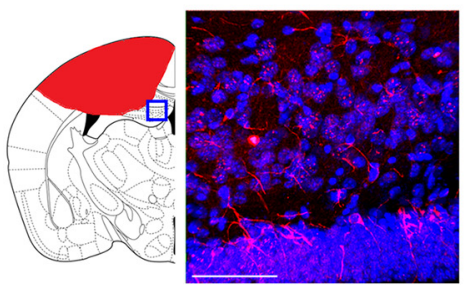

Vehicle
BrdU Dcx DAPI

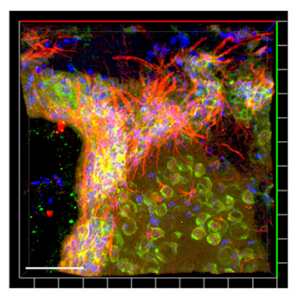

CR2Crry

Dcx
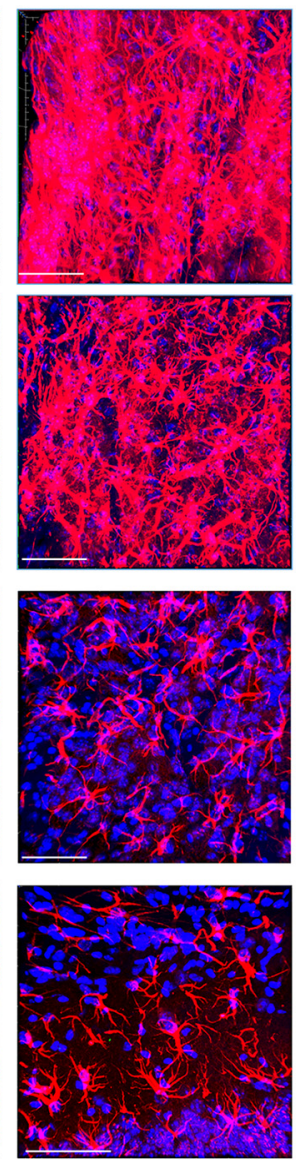

CR2Crry

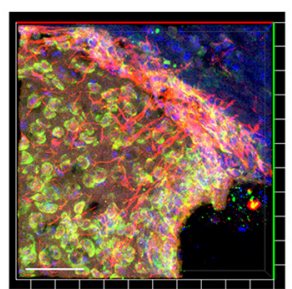

CR2fH

DAPI
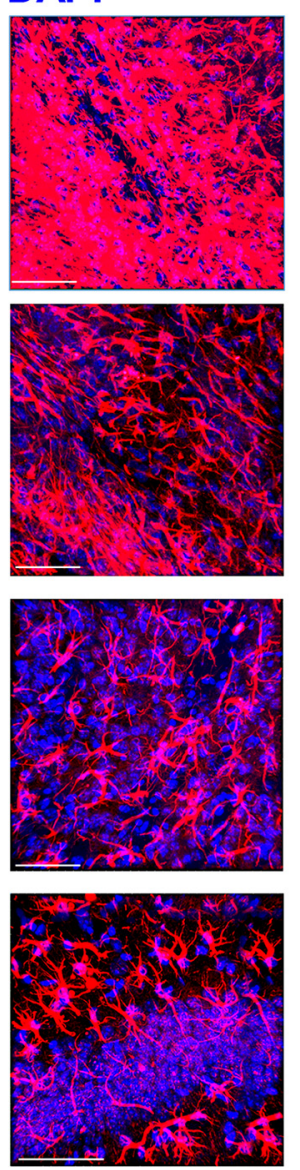

CR2fH

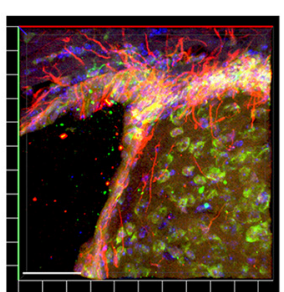

CR2CD59
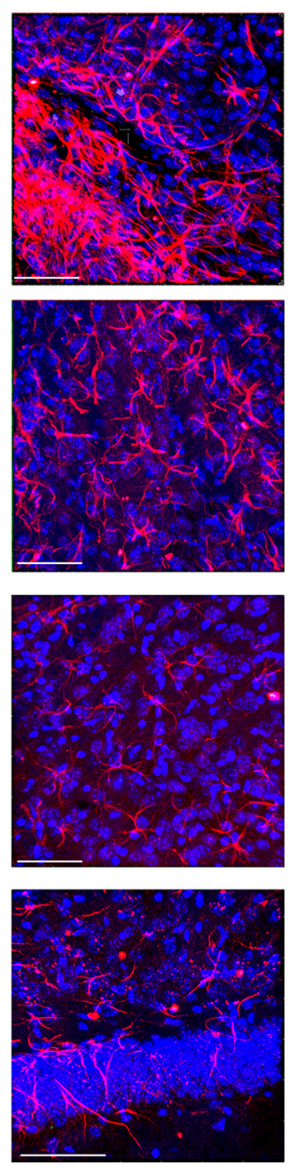

CR2CD59

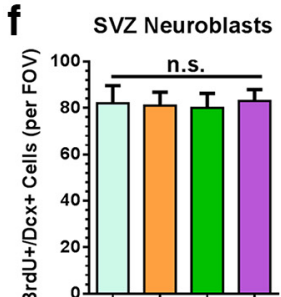

d Lesional Neuroblasts
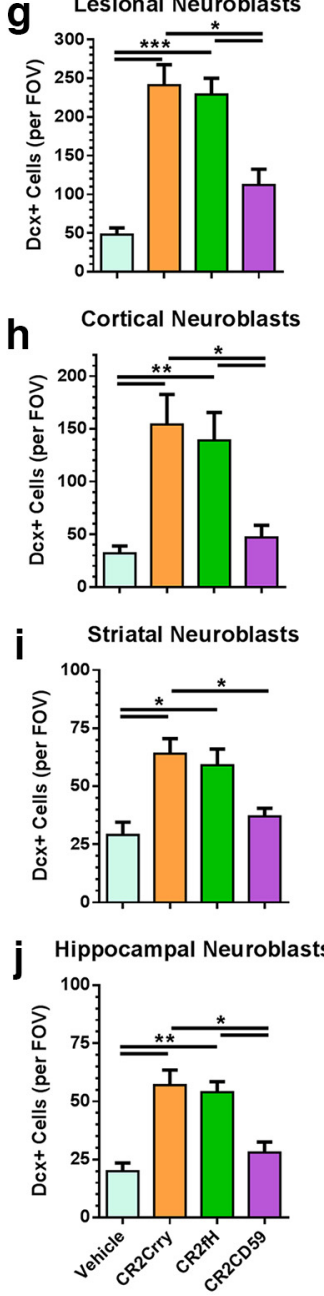

Figure 6. CR2Crry and CR2fH significantly increase neuroblast migration from the SVZ $30 \mathrm{~d}$ after CCl. $\boldsymbol{a}$, IF staining for BrdU $+/ \mathrm{Dcx}+$ cells in and around the subventricular zone $30 \mathrm{~d}$ after CCl showing similar pattern of neurogenesis across the different groups. Scale bars, $50 \mu \mathrm{m}$. $\boldsymbol{b}$-e, Representative Dcx-stained sections of the lesion area ( $\boldsymbol{b}$ ), perilesional cortex (c), basal ganglia (d), and hippocampus (e) showing significantly higher number of $D c x+$ neuroblasts migrating to the lesion site, as well as to perilesional brain in animals treated with CR2Crry and CR2fH compared with both vehicle and CR2CD59. Scale bars, $50 \mu \mathrm{m}$. $\boldsymbol{f}-\boldsymbol{j}$, Quantification of $\boldsymbol{a}$ showing that CR2Crry and CR2fH do not affect SVZ neurogenesis but significantly increase neuronal migration after TBI. One-way ANOVA with Bonferroni's multiple comparisons. $n=5$ /group. ${ }^{*} p<0.05,{ }^{* *} p<0.01,{ }^{* * *} p<0.001$. Error bars $=$ mean \pm SEM.

newly formed neuroblasts. Assessed by the number of SVZ $\mathrm{BrdU}+/ \mathrm{Dcx}+$ neuroblasts, SVZ neurogenesis was not affected by complement inhibition with any of the different targeted inhibitors (Fig. 6a,f). Because neuronal migration from the SVZ to the ipsilateral regions of injury and recovery is key for the neuroreparatory effects of neurogenesis, we investigated neuronal migration to the ipsilateral lesion site (Fig. 6b), and to the perilesional cortex (Fig. $6 c$ ), basal ganglia (Fig. $6 d$ ), and hippocampus (Fig. 6e). Animals treated with CR2Crry or CR2fH had a significantly higher number of migrating Dcx + neuroblasts in the ipsilateral hemisphere compared with vehicle-treated animals, an effect that was not observed in animals treated with CR2CD59 (Fig. 6g-j).

Acute inhibition of $\mathrm{C} 3$ activation, and specifically the ACP, inhibits chronic neurodegeneration after TBI

We finally assessed whether the chronic neuroinflammation observed in vehicle-treated animals and that is reduced by C3 inhibition is associated with more pronounced neurodegeneration at $30 \mathrm{~d}$ after injury. High resolution IF staining of fields from different stereotactic locations relative to the CCI insult for NeuN and Mac2 was used to assess the relation of microgliosis to neuronal 
a
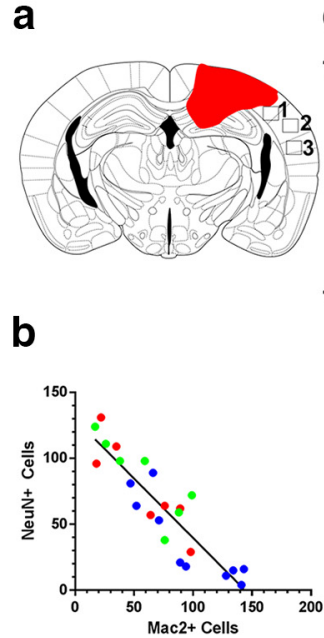

d
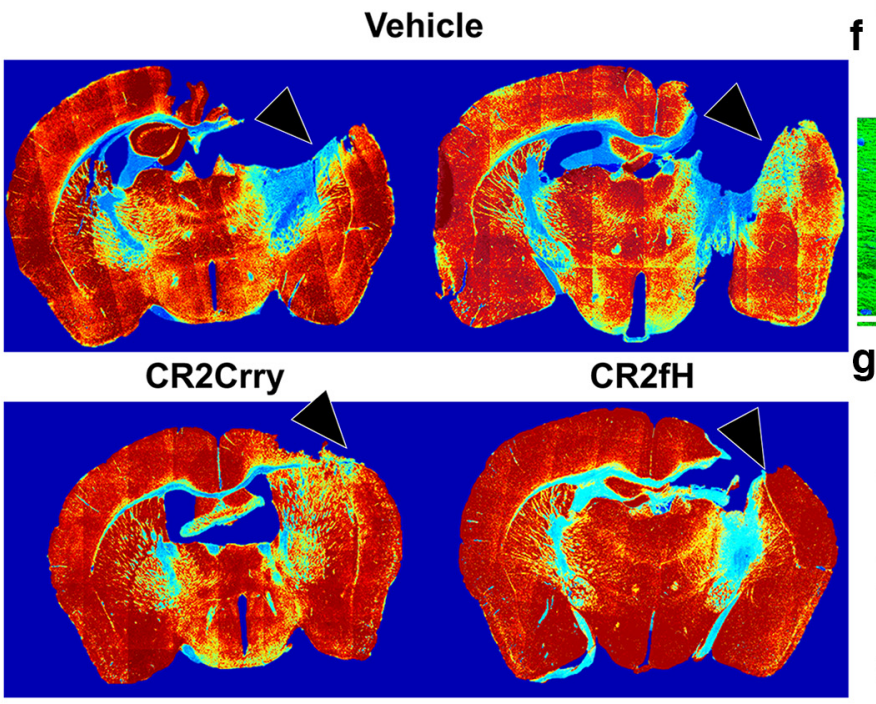

C
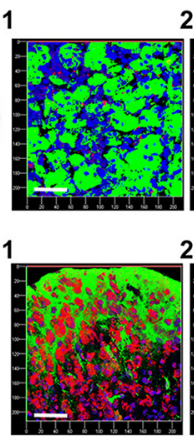

2

2 CR

3
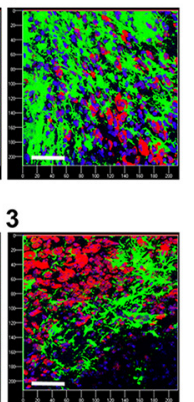

3

2 CR2fH

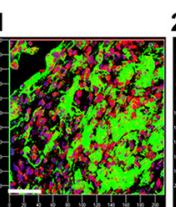

DAPI

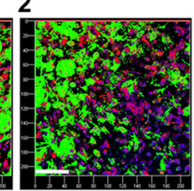

Mac2 f e

$\begin{array}{lll}\text { DAPI } & \text { MAP2 } & \text { C3d } \\ & \text { Vehicle }\end{array}$

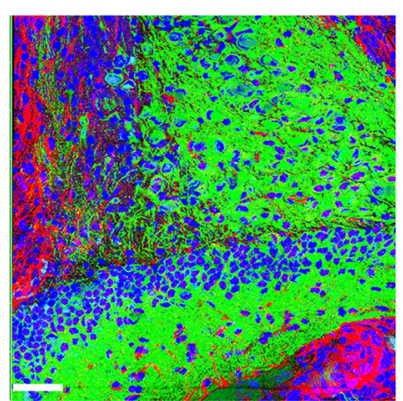

CR2Crry
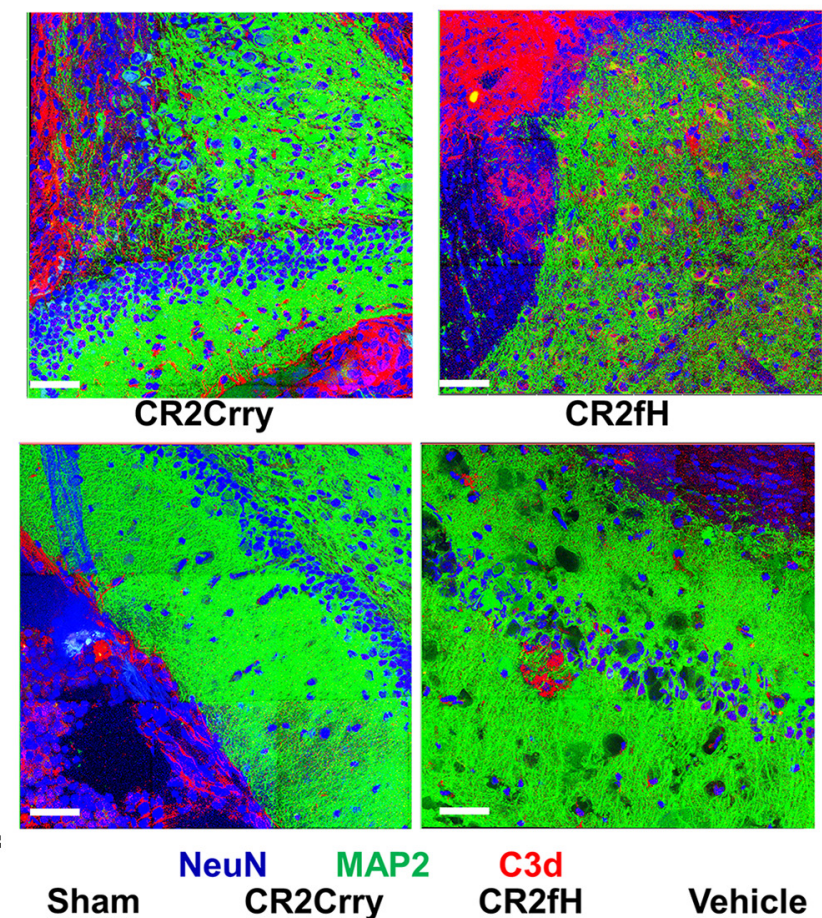

CR2fH

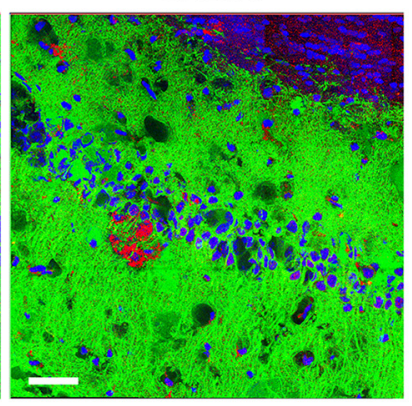

C3d
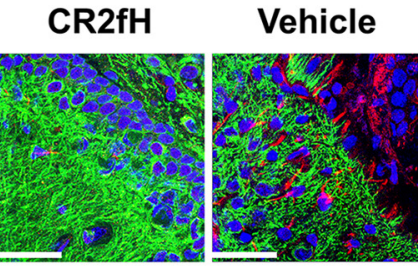

Dendritic Arborization (MAP2+ Fibers)

h

C3d+ Neurons

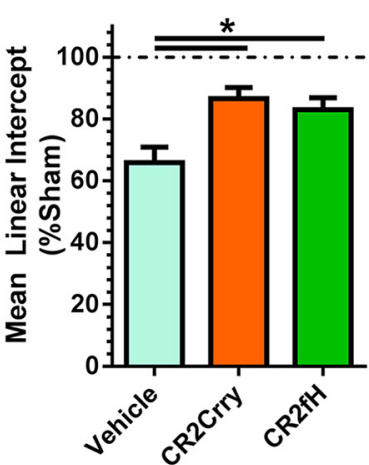

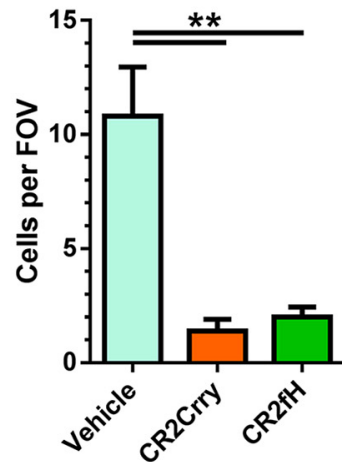

Figure 7. Interaction between chronic neuroinflammation and neurodegenerative processes after CCl. $\boldsymbol{a}$, Representative Nissl stained brain section showing the location of field selection from the perilesional brain for immunostaining shown in this figure. $\boldsymbol{b}$, Correlation between the number of $\mathrm{NeuN}+$ neurons and the number of Mac2 + microglia in perilesional microscopic fields showing a significant negative correlation. Pearson correlation $R^{2}=0.813, p<0.0001$. Blue dots, Vehicle controls; red dots, CR2Crry; green dots, CR2fH. c, Representative high resolution fields showing higher number of $\mathrm{Mac2}+$ cells and lower numbers of NeuN + neurons in the perilesional cortex in vehicle controls compared with CR2fH or CR2Crry-treated animals. Scale bars, $50 \mu \mathrm{m}$. $\boldsymbol{d}$, Heat map of intensity of PSD95 staining in full brain sections from the different treatment groups. $\boldsymbol{e}$, Super-resolution IF staining of dendritic arborization (MAP2, green) and complement deposition (C3d, red) in perilesional hippocampal sections showing significantly higher complement deposition on neurons and less elaborate dendritic arborization in vehicle controls compared with CR2Crry or CR2fH-treated animals. $f$, Higher-magnification fields of e stained for MAP2, NeuN, and C3d showing significantly higher deposition of C3d and loss of dendrites in vehicle compared with sham and to CR2Crry and CR2fH-treated mice. $\boldsymbol{g}, \boldsymbol{h}$, Quantification of staining in $\boldsymbol{e}$ and $\boldsymbol{f}$. One-way ANOVA with Bonferroni's multiple comparisons. $n=5 /$ group. ${ }^{*} p<0.05$, ${ }^{* *} p<0.01$. Error bars $=$ mean \pm SEM.

density in the peri-injury brain (Fig. 7a,b). Vehicle-treated animals showed significantly lower neuronal density in the peri-injury brain compared with CR2Crry or CR2fH-treated mice, with an associated significantly higher density of Mac2 + cells (Fig. 7c). A significant negative correlation was observed between the number of
$\mathrm{Mac} 2+$ cells and the number of NeuN + cells per field indicating that microgliosis is associated with degenerative loss of neurons in the perilesional brain (Fig. 7b). Supporting these findings, immunostaining for PSD95, a marker of synaptic density, showed a significant loss of synaptic density in the perilesional basal ganglia 
a

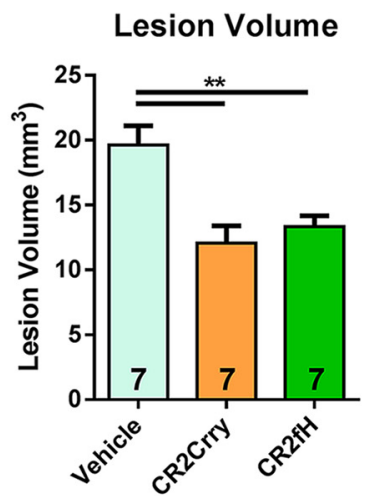

C

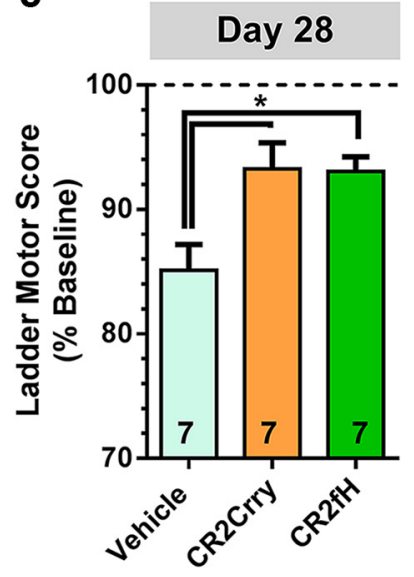

b

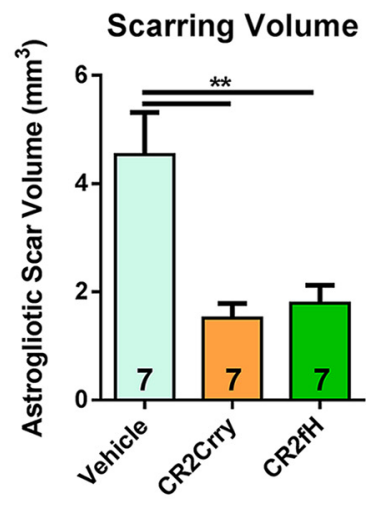

d

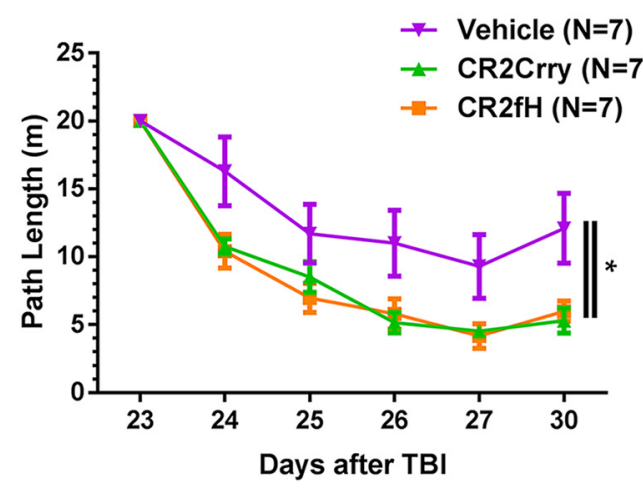

demonstrate a relatively long window of efficacy of these inhibitors and enhance their potential utility in clinical settings.

\section{Discussion}

Previous studies implicated the MAC as a key player in promoting pathology after TBI, and specific inhibition of the MAC has been regarded as a therapeutic approach to reduce post-TBI neuropathology. However, most previous studies only investigated the role of complement in acute outcomes after TBI (Kaczorowski et al., 1995; Stahel et al., 1998, 2009; Leinhase et al., 2006a,b, 2007; Fluiter et al., 2014; Ruseva et al., 2015). Here, we investigated the role of different complement pathways and the relative contribution of the MAC versus upstream activation products on both acute and chronic neurodegeneration after TBI. We confirmed previous data showing a role for the MAC in mediating acute injury after TBI. However, we found it is necessary to inhibit upstream at $\mathrm{C} 3$ activation to prevent the amplification of a feedforward mechanism of cerebral neuroinflammation that persists throughout the chronic phase ( $30 \mathrm{~d}$ after injury). Inhibition of $\mathrm{C} 3$ activation, but not of MAC formation, achieved sustained neuroprotection (mechanism illustrated in Fig. 9). Furthermore, the fact that CR2Crry and CR2fH provided similar levels of protection indicate an important role for the ACP in TBI pathology.

Complement activation results in the generation of complement opsonins (C3 degradation products), anaphylatoxins (C3a and $\mathrm{C} 5 \mathrm{a}$ ), and the MAC. These compo-

and cortex of control animals, with the amount of loss significantly reduced in animals treated with CR2Crry or CR2fH (Fig. $7 d$ ). We also assessed the extent of dendritic arborization in relation to areas of chronic complement activation in the perilesional brain. Vehicle-treated animals showed significant complement deposition in the perilesional brain that colocalized with the neuronal marker NeuN, which was associated with significant loss of dendritic arborization assessed by MAP2 immunostaining. Animals treated with CR2fH or CR2Crry showed significantly less C3d deposition on neurons, as well as more elaborate dendritic arborization compared with vehicle controls (Fig. $7 e-h$ ).

\section{Delayed inhibition of C3 activation is ameliorates motor and cognitive deficits, and improves histopathological outcomes after TBI}

To determine whether the effects or CR2fH or CR2Crry are preserved when administered at a later time after TBI, we administered a single dose at $12 \mathrm{~h}$ after CCI, and assessed outcomes over $30 \mathrm{~d}$. There was a significant improvement in motor and cognitive deficits in animals treated with either CR2Crry or CR2fH after CCI compared with vehicle, as assessed by the ladder rung task (Fig. $8 a$ ) and the Barnes maze task (Fig. $8 b$ ). In addition, both inhibitors were associated with a significant reduction in lesion expansion and gliosis that mimic, albeit to a lesser extent, the effect of earlier administration of inhibitors (Fig. $8 c, d$ ). These findings nents have been show to contribute to different aspects of neuropathology after brain injury (for review, see Alawieh et al., 2015a). Complement opsonins can tag stressed and salvageable neurons for phagocytosis by microglia, mediate phagocytic synaptic loss, and activate microglia and other resident and infiltrating immune cells. Complement anaphylatoxins recruit and activate immune cells, whereas the MAC can contribute to both cell lysis and exacerbation of excitotoxic insult to neurons (for review, see Alawieh and Tomlinson, 2016). Previous work has shown that inhibition of C3 activation at the C3-convertase level (Kaczorowski et al., 1995; Leinhase et al., 2006a), inhibition of the ACP (Leinhase et al., 2006b, 2007), or inhibition of the MAC (Stahel et al., 2009; Ruseva et al., 2015) can all lead to acute neuroprotection after TBI. However, these studies did not assess the role of complement on aspects of neuropathology and functional recovery chronically after TBI.

In a previous work, inhibition of MAC using CD59-2a-CRIg, an injury-site targeted MAC inhibitor, resulted in reduction of acute neuronal loss, inhibition of acute microglial activation, inhibition of mitochondrial stress and improved recovery $3 \mathrm{~d}$ after injury (Ruseva et al., 2015). However, here we report that the neuroprotective effects of MAC inhibition are not sustained chronically after TBI, and our data indicate a role for upstream complement activation products, such as $\mathrm{C} 3$ opsonins and anaphylatoxins, in chronic neurodegeneration after TBI. Supporting 


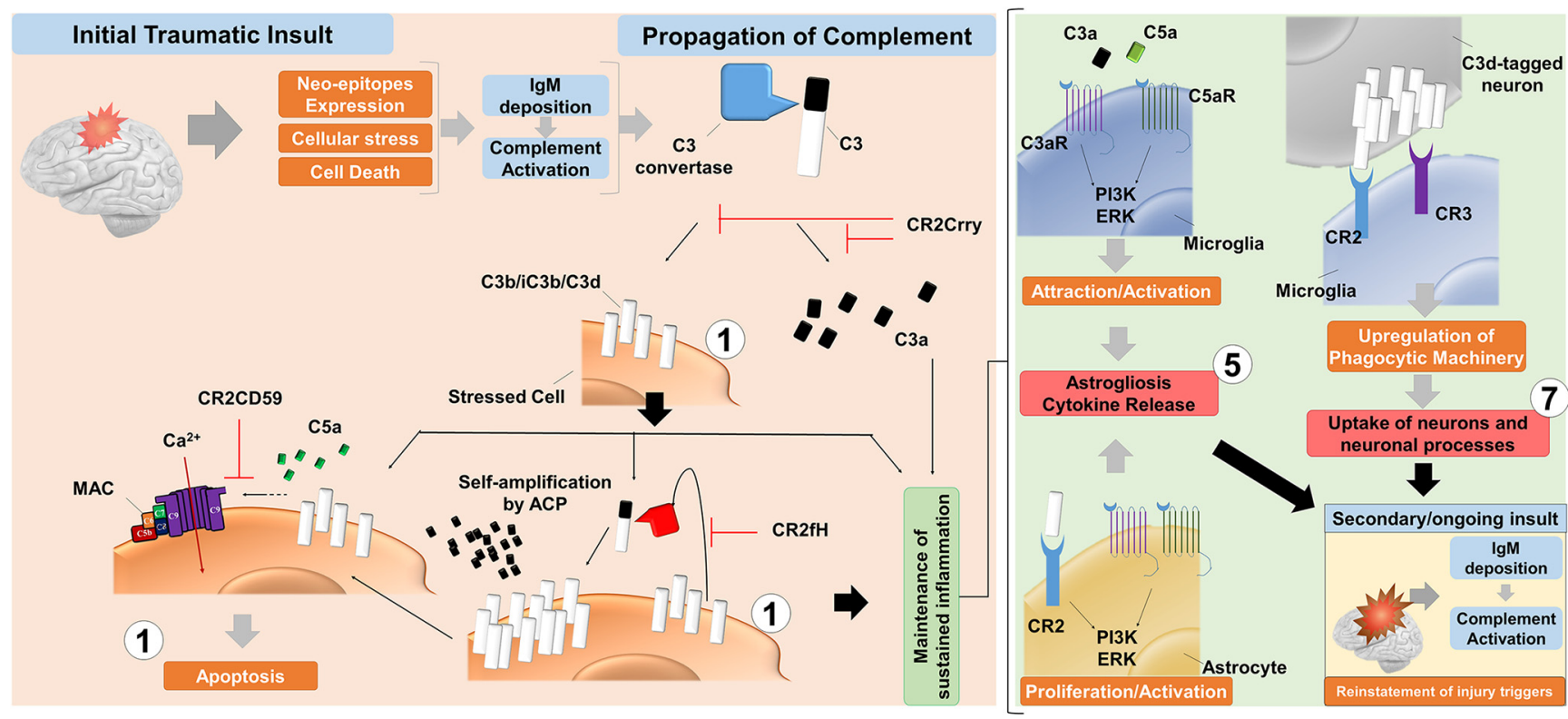

Figure 9. Interaction of different complement activation products with post-TBI pathology. Numbers in circles indicate the reference to figures that provide evidence on the associated step.

this conclusion, previous studies have reported sustained activation of complement 3-7 d after TBI, including elevated anaphylatoxin levels and increased C3d and MAC deposition (Leinhase et al., 2006a,b, 2007; Stahel et al., 2009). In fact, the work of Ruseva et al. (2015) also demonstrated that inhibition of the MAC by CD59-2a-CRIg did not interrupt C3d deposition, and we show here that $\mathrm{C} 3 \mathrm{~d}$ deposition continues for at least $30 \mathrm{~d}$ after TBI, even in the context of MAC inhibition. This chronic C3d deposition is associated with a potent neuroinflammatory response in the brain that interrupts chronic motor and cognitive recovery after TBI, and results in increased microgliosis and tissue damage. It is possible that sustained C3 cleavage may be associated with sustained MAC deposition that drives the pathology; however, we observed only low levels of MAC deposition at $30 \mathrm{~d}$ after TBI in vehicle-treated animals, which was not affected by the different treatments. A possibility is that opsonins and anaphylatoxins contribute to chronic neuroinflammation in a MAC-independent manner by directly activating microglia and immune cells.

CR2-mediated targeting has been previously characterized in the context of ischemic stroke, and has been shown to specifically target sites of C3d deposition in the brain. CR2-targeted complement inhibitors have also been shown to transiently inhibit complement without interrupting complement activation beyond the acute phase (Alawieh et al., 2015b). This controls the duration of complement inhibition and prevents inhibition of complement in recovery, where complement may be important for clearance of debris and neuroregeneration after the acute phase. In addition, the specificity of C3d deposition to sites of injury limits systemic adverse effects of complement inhibition, which could otherwise increase susceptibility to infection, a major concern for TBI patients. A previous strategy described above to deliver a complement inhibitor to the injured brain was via a CRIg-CD59 fusion protein (CD59-2a-CRIg). The CRIg moiety targets sites of C3b deposition (Ruseva et al., 2015). Although C3b and C3d are both cleavage fragments of $\mathrm{C} 3$ breakdown and are specific markers of sites of injury, CR2 recognizes both iC $3 \mathrm{~b}$ and the longerlived fragment C3d, whereas CRIg recognizes only iC3b (Carroll, 1998; Janeway et al., 2001; Holers and Boackle, 2004; Morgan,
2013). Thus, there is likely a very different pharmacodynamic of complement inhibition depending on the targeting strategy used, and it is difficult to predict how this would translate to therapeutic efficacy since it is not clear how long $\mathrm{C} 3$ activation needs to be inhibited to optimally break the cycle leading to chronic inflammation.

To establish the therapeutic efficacy of our strategy, we used a battery of cognitive and motor tasks to assess different aspects of recovery after CCI. Since complement has been implicated in neuronal regeneration and rewiring after brain injury (for review, see Alawieh et al., 2015a), assessment of the impact of complement inhibition on neurogenesis, neuroblast migration, neuronal rewiring and synaptic plasticity, and cognitive recovery beyond the acute phase is needed; these assessments can establish whether acute neuroprotection comes at the cost of inhibition of regeneration. Pan inhibition of C3 activation (CR2Crry) or inhibition of C3 amplification (CR2fH) resulted in a sustained motor and cognitive improvement for $28 \mathrm{~d}$ after TBI compared with vehicle controls. This was associated with a significant increase in neuroblast migration from the subventricular zone and enhanced dendritic and synaptic density in the perilesional brain. We note that these data do not alone provide evidence of a link between complement and neurogenesis after TBI, and many aspects of this response remain uncharacterized; however, the data do indicate that acute and transient inhibition of complement by either CR2Crry or CR2fH is not associated with inhibition of regenerative processes, which is a consideration for potential translation. We demonstrate the presence of a robust neuroinflammatory response in the perilesional brain that is sustained chronically after TBI, and that is associated by ongoing complement deposition, microgliosis, and astrogliosis, leading to continuous loss of neurons and neuronal connections in the perilesional area. In addition, sustained neuroinflammation can also create a negative drive on neurogenesis and neuroblast migration, which may explain the suppression of neuroblast migration in animals treated with vehicle or CR2CD59 compared with CR2Crry or CR2fH. Our data demonstrate that inhibition of complement activation resulted in suppression of microgliosis and astrogliosis after TBI, and was associated with improved cognitive and motor performance. Nevertheless, these findings need to be put in the context of prior work on the neuropathology of 
TBI that has shown that a multitude of inflammatory cascades are triggered after the initial injury, and include components of innate and adaptive immunity (Amor and Woodroofe, 2014). Complement activation, one of the early danger-sensing systems in response to injury, is one of multiple mechanisms and does not alone represent the full repertoire of neuroinflammatory responses after TBI. However, the fact that complement inhibition significantly reduced neuroinflammation and functional deficits after TBI indicate that complement is an important contributor to secondary injury, although the pathophysiologic process of complement activation is not alone likely to explain all functional and histopathological deficits after TBI. Thus, a limitation of this work is the focus on complement, and future work investigating a combination of complement inhibition with other modulators of inflammation may identify an optimal strategy to improve recovery after TBI (Xiong et al., 2013; Amor and Woodroofe, 2014).

In this study, we used the CCI model of TBI that reflects multiple aspects of the histopathological and functional changes that occur after human TBI, but it uses different injury biomechanics consisting of a craniotomy followed by dural impact. Thus, CCI has relatively lower fidelity in modeling concussion and traumatic axonal injury compared with weight drop and other closed head injury models, although it is more reliable in modeling other pathophysiological changes in TBI such as contusions and traumatic brain hemorrhages. As reviewed by Xiong et al. (2013), current animal models of TBI, including CCI, still provide an incomplete reflection of the pathophysiological processes that occur in human TBI, which represents a challenge and a limitation in preclinical research on brain injury. Thus, although this work demonstrates the efficacy of translational complement inhibitors in one murine model of TBI, more work will be required before humanized counterparts could be investigated in clinical trials, such as evaluation in different TBI models and across different animal species. In addition to the limitations of preclinical models, our work did not address gender, comorbidities, and age-related effects on outcome of complement inhibition in TBI, and did not investigate the effect of complement modulation with different TBI severity measures. However, the work here still addresses important aspects of preclinical TBI research, including targeting anti-inflammatory therapy specifically to the site of injury and thus limiting systemic effects, the administration of inhibitors up to $12 \mathrm{~h}$ after the onset of TBI rather than before or just after insult, the assessment of outcomes at least 4 weeks after TBI, and the use of a diverse battery of motor and cognitive tasks. Also of note, recent data show that a humanized version of CR2 $\mathrm{fH}$ is safe and non-immunogenic in human subjects (Risitano et al., 2015).

\section{References}

Alawieh A, Tomlinson S (2016) Injury site-specific targeting of complement inhibitors for treating stroke. Immunol Rev 274:270-280. CrossRef Medline

Alawieh A, Narang A, Tomlinson S (2015a) Complementing regeneration. Oncotarget 6:21769-21770. CrossRef Medline

Alawieh A, Elvington A, Zhu H, Yu J, Kindy MS, Atkinson C, Tomlinson S (2015b) Modulation of post-stroke degenerative and regenerative processes and subacute protection by site-targeted inhibition of the alternative pathway of complement. J Neuroinflammation 12:247. CrossRef Medline

Amor S, Woodroofe MN (2014) Innate and adaptive immune responses in neurodegeneration and repair. Immunology 141:287-291. CrossRef Medline

Arciniegas DB, Held K, Wagner P (2002) Cognitive impairment following traumatic brain injury. Curr Treat Options Neurol 4:43-57. CrossRef Medline

Atkinson C, Song H, Lu B, Qiao F, Burns TA, Holers VM, Tsokos GC, Tom- linson S (2005) Targeted complement inhibition by C3d recognition ameliorates tissue injury without apparent increase in susceptibility to infection. J Clin Invest 115:2444-2453. CrossRef Medline

Bellander BM, Singhrao SK, Ohlsson M, Mattsson P, Svensson M (2001) Complement activation in the human brain after traumatic head injury. J Neurotrauma 18:1295-1311. CrossRef Medline

Bellander BM, Olafsson IH, Ghatan PH, Bro Skejo HP, Hansson LO, Wanecek M, Svensson MA (2011) Secondary insults following traumatic brain injury enhance complement activation in the human brain and release of the tissue damage marker S100B. Acta Neurochir (Wien) 153: 90-100. CrossRef Medline

Blennow K, Hardy J, Zetterberg H (2012) The neuropathology and neurobiology of traumatic brain injury. Neuron 76:886-899. CrossRef Medline

Carroll MC (1998) CD21/CD35 in B cell activation. Semin Immunol 10: 279-286. CrossRef Medline

Fluiter K, Opperhuizen AL, Morgan BP, Baas F, Ramaglia V (2014) Inhibition of the membrane attack complex of the complement system reduces secondary neuroaxonal loss and promotes neurologic recovery after traumatic brain injury in mice. J Immunol 192:2339-2348. CrossRef Medline

Fox GB, Fan L, Levasseur RA, Faden AI (1998) Sustained sensory/motor and cognitive deficits with neuronal apoptosis following controlled cortical impact brain injury in the mouse. J Neurotrauma 15:599-614. CrossRef Medline

Hicks RR, Keeling KL, Yang MY, Smith SA, Simons AM, Kotwal GJ (2002) Vaccinia virus complement control protein enhances functional recovery after traumatic brain injury. J Neurotrauma 19:705-714. CrossRef Medline

Holers VM, Boackle SA (2004) Complement receptor 2 and autoimmunity. Curr Dir Autoimmun 7:33-48. Medline

Huang Y, Qiao F, Atkinson C, Holers VM, Tomlinson S (2008) A novel targeted inhibitor of the alternative pathway of complement and its therapeutic application in ischemia/reperfusion injury. J Immunol 181:8068 8076. CrossRef Medline

Janeway CA, Travers P, Walport M (2001) The complement system and innate immunity, Ed 5. New York: Garland Science.

Kaczorowski SL, Schiding JK, Toth CA, Kochanek PM (1995) Effect of soluble complement receptor-1 on neutrophil accumulation after traumatic brain injury in rats. J Cereb Blood Flow Metab 15:860-864. CrossRef Medline

Kossmann T, Stahel PF, Morganti-Kossmann MC, Jones JL, Barnum SR (1997) Elevated levels of the complement components C3 and factor B in ventricular cerebrospinal fluid of patients with traumatic brain injury. J Neuroimmunol 73:63-69. CrossRef Medline

Kumar A, Loane DJ (2012) Neuroinflammation after traumatic brain injury: opportunities for therapeutic intervention. Brain Behav Immun 26: 1191-1201. CrossRef Medline

Leinhase I, Schmidt OI, Thurman JM, Hossini AM, Rozanski M, Taha ME, Scheffler A, John T, Smith WR, Holers VM, Stahel PF (2006a) Pharmacological complement inhibition at the $\mathrm{C} 3$ convertase level promotes neuronal survival, neuroprotective intracerebral gene expression, and neurological outcome after traumatic brain injury. Exp Neurol 199:454464. CrossRef Medline

Leinhase I, Holers VM, Thurman JM, Harhausen D, Schmidt OI, Pietzcker M, Taha ME, Rittirsch D, Huber-Lang M, Smith WR, Ward PA, Stahel PF (2006b) Reduced neuronal cell death after experimental brain injury in mice lacking a functional alternative pathway of complement activation. BMC Neurosci 7:55. CrossRef Medline

Leinhase I, Rozanski M, Harhausen D, Thurman JM, Schmidt OI, Hossin AM, Taha ME, Rittirsch D, Ward PA, Holers VM, Ertel W, Stahel PF (2007) Inhibition of the alternative complement activation pathway in traumatic brain injury by a monoclonal anti-factor B antibody: a randomized placebo-controlled study in mice. J Neuroinflammation 4:13. CrossRef Medline

Longhi L, Perego C, Ortolano F, Zanier ER, Bianchi P, Stocchetti N, McIntosh TK, De Simoni MG (2009) C1-inhibitor attenuates neurobehavioral deficits and reduces contusion volume after controlled cortical impact brain injury in mice. Crit Care Med 37:659-665. CrossRef Medline

Marshall KM, He S, Zhong Z, Atkinson C, Tomlinson S (2014) Dissecting the complement pathway in hepatic injury and regeneration with a novel protective strategy. J Exp Med 211:1793-1805. CrossRef Medline

Metz GA, Whishaw IQ (2009) The ladder rung walking task: a scoring system and its practical application. J Vis Exp 28:e1204. CrossRef Medline 
Morgan BP (2013) Complement. In: Fundamental immunology, Ed 7 (Paul WE, ed). Philadelphia: Lippincott Williams and Wilkins.

Neher MD, Weckbach S, Flierl MA, Huber-Lang MS, Stahel PF (2011) Molecular mechanisms of inflammation and tissue injury after major trauma: is complement the "bad guy"? J Biomed Sci 18:90. CrossRef Medline

Patil SS, Sunyer B, Höger H, Lubec G (2009) Evaluation of spatial memory of C57BL/6J and CD1 mice in the Barnes maze, the multiple T-maze and in the morris water maze. Behav Brain Res 198:58-68. CrossRef Medline

Rahpeymai Y, Hietala MA, Wilhelmsson U, Fotheringham A, Davies I, Nilsson AK, Zwirner J, Wetsel RA, Gerard C, Pekny M, Pekna M (2006) Complement: a novel factor in basal and ischemia-induced neurogenesis. EMBO J 25:1364-1374. CrossRef Medline

Rancan M, Morganti-Kossmann MC, Barnum SR, Saft S, Schmidt OI, Ertel W, Stahel PF (2003) Central nervous system-targeted complement inhibition mediates neuroprotection after closed head injury in transgenic mice. J Cereb Blood Flow Metab 23:1070-1074. CrossRef Medline

Rich MC, Keene CN, Neher MD, Johnson K, Yu ZX, Ganivet A, Holers VM, Stahel PF (2016) Site-targeted complement inhibition by a complement receptor 2-conjugated inhibitor (mTT30) ameliorates post-injury neuropathology in mouse brains. Neurosci Lett 617:188-194. CrossRef Medline

Risitano AM, Storek M, Sahelijo L, Doyle M, Dai Y, Weitz IC, Marsh JC, Elebute M, O'Connell CL, Kulasekararaj AG (2015) Safety and pharmacokinetics of the complement inhibitor TT30 in a phase I trial for untreated PNH patients. Blood 126:2137.

Ruseva MM, Ramaglia V, Morgan BP, Harris CL (2015) An anticomplement agent that homes to the damaged brain and promotes recovery after traumatic brain injury in mice. Proc Natl Acad Sci U S A 112:1431914324. CrossRef Medline

Sewell DL, Nacewicz B, Liu F, Macvilay S, Erdei A, Lambris JD, Sandor M, Fabry Z (2004) Complement C3 and C5 play critical roles in traumatic brain cryoinjury: blocking effects on neutrophil extravasation by $\mathrm{C} 5 \mathrm{a}$ receptor antagonist. J Neuroimmunol 155:55-63. CrossRef Medline
Smith DH, Soares HD, Pierce JS, Perlman KG, Saatman KE, Meaney DF, Dixon CE, McIntosh TK (1995) A model of parasagittal controlled cortical impact in the mouse: cognitive and histopathologic effects. J Neurotrauma 12:169-178. CrossRef Medline

Song H, He C, Knaak C, Guthridge JM, Holers VM, Tomlinson S (2003) Complement receptor 2-mediated targeting of complement inhibitors to sites of complement activation. J Clin Invest 111:1875-1885. CrossRef Medline

Stahel PF, Morganti-Kossmann MC, Kossmann T (1998) The role of the complement system in traumatic brain injury. Brain Res Rev 27:243-256. CrossRef Medline

Stahel PF, Morganti-Kossmann MC, Perez D, Redaelli C, Gloor B, Trentz O, Kossmann T (2001) Intrathecal levels of complement-derived soluble membrane attack complex (sC5b-9) correlate with blood-brain barrier dysfunction in patients with traumatic brain injury. J Neurotrauma 18: 773-781. CrossRef Medline

Stahel PF, Flierl MA, Morgan BP, Persigehl I, Stoll C, Conrad C, Touban BM, Smith WR, Beauchamp K, Schmidt OI, Ertel W, Leinhase I (2009) Absence of the complement regulatory molecule CD59a leads to exacerbated neuropathology after traumatic brain injury in mice. J Neuroinflammation 6:2. CrossRef Medline

Türeyen K, Vemuganti R, Sailor KA, Dempsey RJ (2004) Infarct volume quantification in mouse focal cerebral ischemia: a comparison of triphenyltetrazolium chloride and cresyl violet staining techniques. J Neurosci Methods 139:203-207. CrossRef Medline

Xiong Y, Mahmood A, Chopp M (2013) Animal models of traumatic brain injury. Nat Rev Neurosci 14:128-142. CrossRef Medline

Zhang L, Schallert T, Zhang ZG, Jiang Q, Arniego P, Li Q, Lu M, Chopp M (2002) A test for detecting long-term sensorimotor dysfunction in the mouse after focal cerebral ischemia. J Neurosci Methods 117:207-214. CrossRef Medline 